Prepared for the U.S. Department of Energy

under Contract DE-AC05-76RL01830

\title{
Progress on Generic Phase-Field Method Development
}

SB Biner

MR Tonks

PC Millett

$\mathrm{Y} \mathrm{Li}$

$\mathrm{SHu}$

F Gao
$X$ Sun

D Schwen

E Martinez

A Caro

D Anderson

September 2012

Pacific Northwest

NATIONAL LABORATORY

Proudly Operated by Battelle Since 1965 



\title{
DISCLAIMER
}

This report was prepared as an account of work sponsored by an agency of the United States Government. Neither the United States Government nor any agency thereof, nor Battelle Memorial Institute, nor any of their employees, makes any warranty, express or implied, or assumes any legal liability or responsibility for the accuracy, completeness, or usefulness of any information, apparatus, product, or process disclosed, or represents that its use would not infringe privately owned rights. Reference herein to any specific commercial product, process, or service by trade name, trademark, manufacturer, or otherwise does not necessarily constitute or imply its endorsement, recommendation, or favoring by the United States Government or any agency thereof, or Battelle Memorial Institute. The views and opinions of authors expressed herein do not necessarily state or reflect those of the United States Government or any agency thereof.

\author{
PACIFIC NORTHWEST NATIONAL LABORATORY \\ operated by \\ BATTELLE \\ for the \\ UNITED STATES DEPARTMENT OF ENERGY \\ under Contract DE-AC05-76RL01830
}

Printed in the United States of America
Available to DOE and DOE contractors from the Office of Scientific and Technical Information,
P.O. Box 62, Oak Ridge, TN 37831-0062;
ph: (865) 576-8401
fax: $(865)$ 576-5728
email: reports@adonis.osti.gov

\begin{abstract}
Available to the public from the National Technical Information Service, U.S. Department of Commerce, 5285 Port Royal Rd., Springfield, VA 22161 ph: (800) 553-6847 fax: $(703) 605-6900$ email: orders@ntis.fedworld.gov online ordering: http://www.ntis.gov/ordering.htm
\end{abstract}

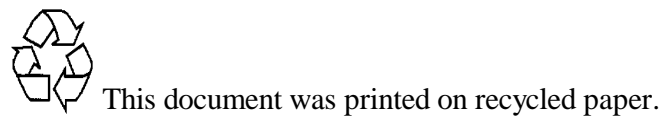




\title{
MILESTONE REPORT:
}

\section{PROGRESS ON GENERIC PHASE-FIELD METHOD DEVELOPMENT}

\author{
S.B. Biner ${ }^{1}$, M.R. Tonks ${ }^{1}$, P.C. Millett ${ }^{1}$

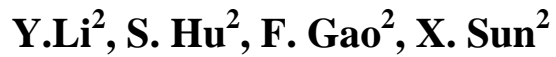 \\ D. Schwen ${ }^{3}$, E. Martinez ${ }^{3}$, A. Caro ${ }^{3}$, D. Anderson ${ }^{3}$ \\ ${ }^{1}$ Idaho National Laboratory \\ 2 Pacific Northwest National Laboratory \\ ${ }^{3}$ Los Alamos National Laboatory
}

September 30, 2012

\begin{abstract}
In this report, we summarize our current collobarative efforts, involving three national laboratories: Idaho National Laboratory (INL), Pacific Northwest National Laboratory (PNNL) and Los Alamos National Laboatory (LANL), to develop a computational framework for homogenous and heterogenous nucleation mechanisms into the generic phase-field model. During the studies, the Fe-Cr system was chosen as a model system due to its simplicity and availability of reliable thermodynamic and kinetic data, as well as the range of applications of low-chromium ferritic steels in nuclear reactors. For homogenous nucleation, the relavant parameters determined from atomistic studies were used directly to determine the energy functional and parameters in the phase-field model. Interfacial energy, critical nucleus size, nucleation rate, and coarsening kinetics were systematically examined in two- and three- dimensional models. For the heteregoneous nucleation mechanism, we studied the nucleation and growth behavior of chromium precipitates due to the presence of dislocations. The results demonstrate that both nucleation schemes can be introduced to a phase-field modeling algorithm with the desired accuracy and computational efficiency.
\end{abstract}




\section{TABLE OF CONTENTS}

\section{$\underline{\text { Page Number }}$}

I. Introduction

3

II. Atomistic studies for the analytical calculation of critical size for alpha prime precipitation in Fe-Cr system (LANL)

III. Phase-field model of homogenous nucleation of $\mathrm{Cr}$ precipitates in $\mathrm{Fe}-\mathrm{Cr}$ alloys (PNNL)

IV. Heteregenous nucleation behavior due to the presence of dislocation in $\mathrm{Fe}-\mathrm{Cr}$ system (INL)

V. Concluding remarks

VI. References 


\section{INTRODUCTION}

The phase-field method is gaining popularity to model microstructure evolution under different thermodynamic driving conditions. In most phase-field modeling, the phasefield equations are deterministic with the evolution of the phase-field variables driven by the reduction in the total free energy of an inhomogeneous system. Although phase-field modeling successfully accounts for growth kinetics, there are no efficient models to accurately and efficiently include the nucleation kinetics. This is particularly difficult for the homogenous nucleation behavior due to the short temporal and small spatial scales associated with this nucleation mechanism. Currently, homogeneous nucleation events are handled within the phase-field modeling framework either with the introduction of a Langevin noise term, where the initial state is not too far from the instability temperature or composition, or via explicit nucleation methods based on the classical nucleation theory and Poisson seeding. The advantages and disadvantages of both methods have recently been summarized in [1]. It is now well established that the presence of submicrostructural features, such as dislocations and grain boundaries, provides thermodynamically favorable sites for nucleation and alters the evolution kinetics by providing different diffusional pathways.

In this study, both nucleation mechanisms are incoprated into the phase-field formalism. The Fe-Cr system was choosen as a model system due to its simplicity and availability of reliable thermodynamic and kinetic data, as well as the range of applications of lowchromium ferritic steels in nuclear reactors.

The report is organized in three main sections and a conclusion: In the first section, the details of the studies at the atomistic scale for generation of the necessary parameters used for the homogenous nucleation events are described. The second section follows with the demonstration of a generic phase-field modeling methodology for homogenous nucleation. In the third section, we present the results for heteregenous nucleation and growth behavior of chromium precipates due the presence of dislocations. Finally, we offer our concluding remarks. 


\section{Atomistic studies for the analytical calculation of critical size for $\mathrm{Cr}$ precipitation in the Fe-Cr system}

Fe-Cr systems show a miscibility gap in the bcc solid solution phase that becomes heterogeneous with an $\alpha$-phase (Fe-rich solid solution) and an $\alpha^{\prime}$-phase (Cr-rich solid solution). Only recently has data become available for both the free energy of the solid solution and the interfacial free energy of the Fe-Cr system. Our group obtained the free energy of the solid solution phase using a switching Hamiltonian method in a molecular dynamics simulation with an empirical concentration-dependent embedded atom (CDEAM) potential $[2,3]$. The applicable range of the resulting free energy surface goes from room temperature up to $700 \mathrm{~K}$, well below the Curie temperature. Above this temperature, the model is not accurate as magnetic effects become important, and below it, classical nuclei mechanics is not valid (the Debye temperature of $\mathrm{Fe}$ is $460 \mathrm{~K}$ ).

The interfacial free energy was obtained using variance-constrained semi-grand canonical Monte Carlo (VCSGCMC) calculations by Sadigh and Erhart [4] with the same interatomic potential. The authors observe similar values for the three cubic orientations of the interface, namely (100), (110), and (111), which implies nearly spherical precipitates. Ab initio calculations by Lu et al. $[5,6]$ on the (001) and (110) interfaces support the observed isotropy and offer excellent agreement on the energy values.

When calculating the interfacial free energies, a possible radius dependence was ignored. In practice, the cluster interfacial free energy can strongly depend on cluster radii for small cluster sizes and vary from the bulk values, as calculated by DFT for example.

Due to the low lattice mismatch, the elastic energy contribution in the Fe-Cr system is negligible and omitted in this study, greatly simplifying the derivations of the critical radius and nucleation rate. We note that the framework of classical nucleation theory is strictly valid only in the limit of low supersaturations [7]. Furthermore, the latent heat released during nucleation, which can lead to a local temperature increase, also is ignored. This is justified by the good heat conductivity of the alloy as well as the long timescale of the kinetically limited nucleation processes.

The nucleation treatment in this work uses equilibrium thermodynamics and a steady 
state approximation. This is justified because we are only interested in instantaneous nucleation rates within individual phase-field cells, which by design contain no other nuclei. Growth and coarsening of the resulting precipitates is then treated within the phase-field framework.

The maximal nucleation driving force, obtained using the parallel tangent construction, can vary if the precipitate composition is allowed to deviate from the $\alpha^{\prime}$ equilibrium composition. However, because the solubility of $\mathrm{Fe}$ in $\mathrm{Cr}$ for the potential used in this work, as well as other Fe-Cr potentials $[8,9]$ is low below $700 \mathrm{~K}$, changes in the driving force due to changes in $\alpha^{\prime}$ composition are expected to be small.

The free energy surface $G(x, T)$ as a function of $\mathrm{Cr}$ concentration $x$ and temperature $T$ is given by the following set of equations, where $H_{\chi}$ are the composition-dependent coefficients of a polynomial fit to the enthalpy calculated using molecular dynamics, and $G_{0}$ is the free energy at the switching temperature obtained using the Hamiltonian switching method. All fitting parameters are listed in Table-1, and a plot of the free energy surface is given in Figure 1.

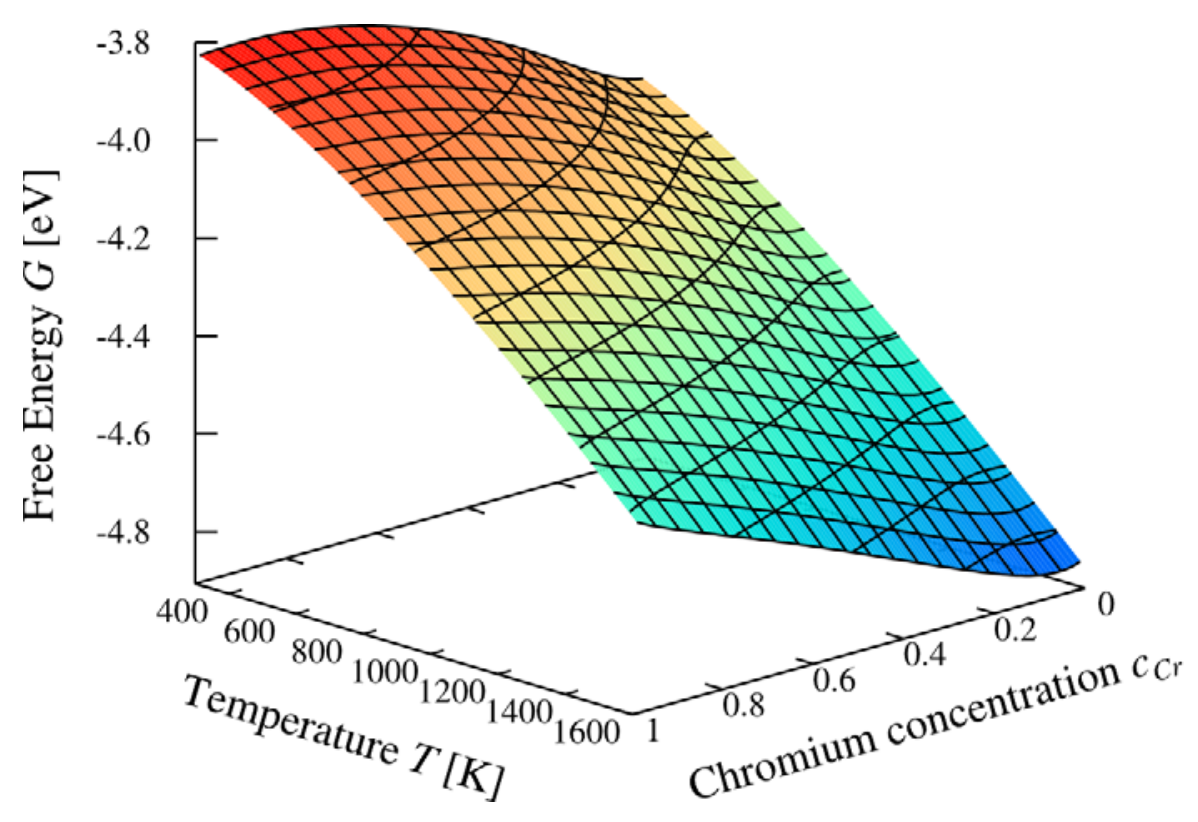

Figure 1. Free energy surface $\mathrm{G}(\mathrm{x}, \mathrm{T})$ 


$$
\begin{aligned}
& H_{a}(x)=x(1-x)\left[\sum_{i=0}^{5} a_{i}(1-2 x)^{i}\right]+a_{6} x+a_{7}(1-x) \\
& H_{\xi \in\{b, c, d\}}(x)=\sum_{i=0}^{3} \xi_{i}(1-x)^{i} \\
& H_{e}(x)=-\frac{H_{a}(x)}{T_{0}}+H_{c}(x) T_{0}+H_{b}(x) \log T_{0}+H_{b}(x) \frac{T_{0}^{2}}{2} \\
& G_{0}(x)=x(1-x)\left[\sum_{i=0}^{5} f_{i}(1-2 x)^{i}\right]+f_{6} x+f_{7}(1-x) \\
& G(x, T)=G_{0}(x) \frac{T}{T_{0}}+H_{a}(x)-H_{b}(x) T \log T-H_{c}(x) T^{2}+H_{d}(x) \frac{T^{3}}{2}+H_{e}(x) T \\
& \quad+T k_{B}(x \log x+(1-x) \log (1-x))
\end{aligned}
$$

The final expression results from applying the Gibbs-Duhem equation and adding the configurational entropy of an ideal solution. The form of the equations closely follows the CALPHAD [10] standard. Although in alloys with a negative heat of formation, short-range order in the solid solution may develop, it has been shown that the random solution approximation still adequately models the free energy and resulting phase diagram [9]. Figure 2 shows the phase diagram for the Fe-Cr system as modeled by our potential constructed using the common tangent method. The solid curve marks the miscibility gap of the Fe-Cr system, while the dashed curve marks the spinodal line (the boundary of the thermodynamically unstable region, given by $\frac{d^{2} G}{d x^{2}}=0$ ). The diamond symbols with error bars indicate the solubility limits obtained by a SGCMC simulation. The shaded region gives a range of possible $\mathrm{Cr}$ solubility limits in $\mathrm{Fe}$ as obtained by Xiong et al. [11], while the triangles are results from cluster expansion (CE) calculations fitted to first principles data [12]. In the validity region of the potential, we observe that the results from the the CD-EAM model agree quite well with the Xiong et al. values and are similar to those given by the CE. The CD-EAM potential is fitted to the mixing enthalpy expressed by a coherent potential approximation obtained by Olsson et al. [13]. The maximum value of this mixing enthalpy is more than $20 \mathrm{meV}$ larger than the one obtained using special quasi-random structures [14]. This difference, and the difference in curvature, partially explains the lower $\mathrm{Cr}$ solubility obtained with the CD-EAM 
compared to the results by Xiong et al. [11].

\begin{tabular}{lrrrrr}
\hline$\xi=$ & $a$ & $b$ & $c$ & $d$ & $f$ \\
\hline$\xi_{0}$ & 0.3856 & 0.0003 & $3.1446 \cdot 10^{-9}$ & $-1.7601 \cdot 10^{-13}$ & 0.3817 \\
$\xi_{1}$ & -0.0973 & $4.6956 \cdot 10^{-5}$ & $-2.2031 \cdot 10^{-8}$ & $5.5788 \cdot 10^{-12}$ & -0.1007 \\
$\xi_{2}$ & -0.0467 & $-4.9588 \cdot 10^{-5}$ & $3.9597 \cdot 10^{-8}$ & $-5.9353 \cdot 10^{-12}$ & -0.0485 \\
$\xi_{3}$ & -0.1945 & $1.1333 \cdot 10^{-5}$ & $-3.0902 \cdot 10^{-8}$ & $1.2285 \cdot 10^{-11}$ & -0.1541 \\
$\xi_{4}$ & -0.1856 & 0.0000 & 0.0000 & 0.0000 & -0.1684 \\
$\xi_{5}$ & -0.0044 & 0.0000 & 0.0000 & 0.0000 & -0.0416 \\
$\xi_{6}$ & -3.8366 & 0.0000 & 0.0000 & 0.0000 & -3.8602 \\
$\xi_{7}$ & -4.1231 & 0.0000 & 0.0000 & 0.0000 & -4.1671 \\
\hline
\end{tabular}

Table-1 Coefficients for the $\mathrm{Fe}-\mathrm{Cr}$ free energy surface given in Equations 1-5.

To obtain an expression for the interfacial free energy, we fit the second degree polynomial $F_{i}[T]=e_{1}-e_{2} T-e_{3} T^{2}$ to the data reported by Sadigh and Erhart [4]. Figure 3 shows both our data and that of Sadigh. The values for the coeffients are $e_{1}=441.4385 \frac{\mathrm{J}}{\mathrm{m}^{2}}, e_{2}=0.1091 \frac{\mathrm{J}}{\mathrm{Km}^{2}}$, and $e_{3}=4.2794 \cdot 10^{-5} \frac{\mathrm{J}}{\mathrm{K}^{2} \mathrm{~m}^{2}}$.

The composition $c_{\alpha}^{\prime}(T)$ of the terminal phase on the Fe-rich side at the temperature $T$ was fit to the calculated phase diagram shown in Figure 2 using a third order polynomial:

$c_{\alpha^{\prime}}(T)=\chi_{0}+\chi_{1} T+\chi_{2} T^{2}+\chi_{3} T^{3}$

with the parameters $\chi_{0}=2.557 \cdot 10^{-2}, \quad \chi_{1}=5.120 \cdot 10^{-5}, \quad \chi_{2}=8.730 \cdot 10^{-9}$, and $\chi_{3}=2.065 \cdot 10^{-11}$. For the Cr-rich side, we assume this composition to be independent of temperature at $c_{\alpha}=0.998$. 


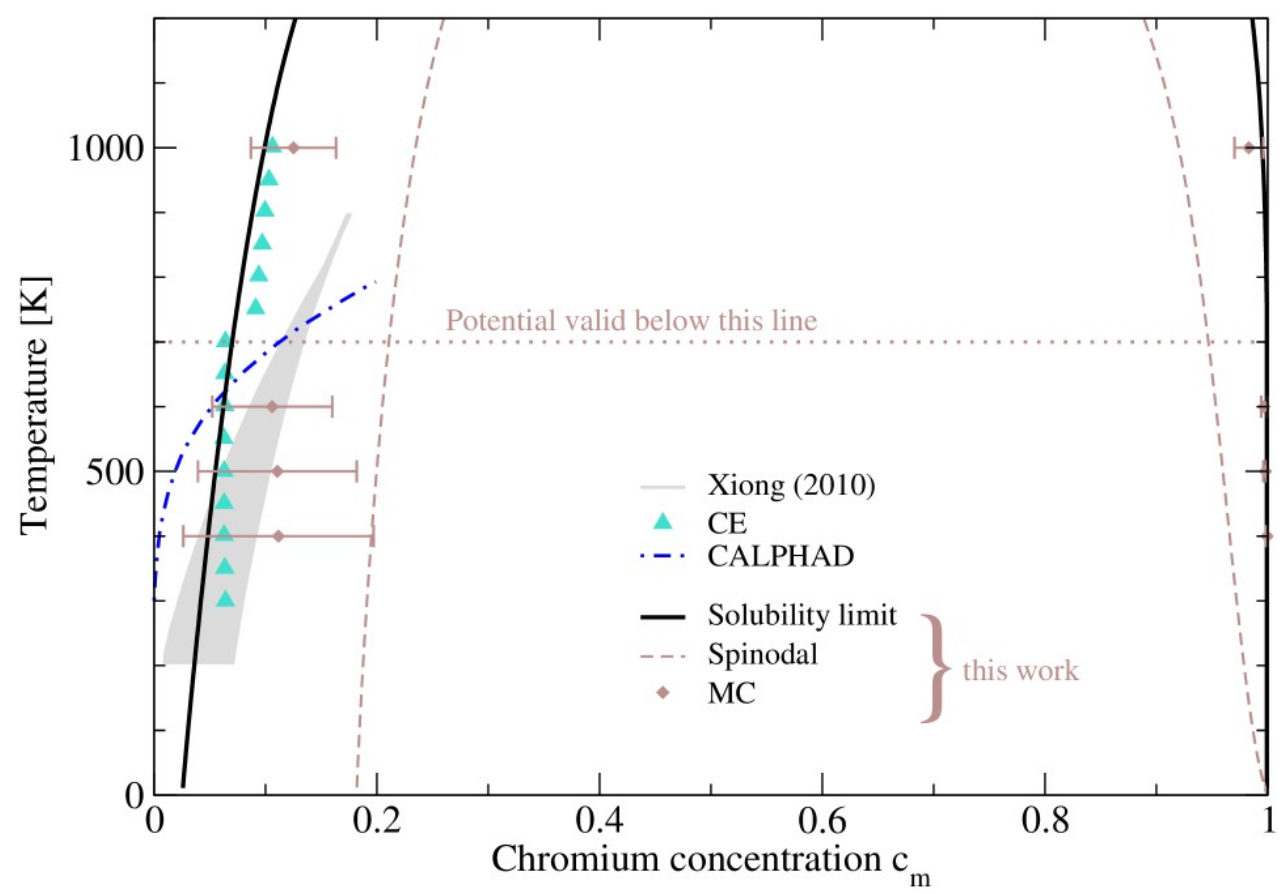

Figure 2. Phase diagram of the Fe-Cr system calculated from the free energy surface. The solid curve marks the miscibility gap of the Fe-Cr system, and the dashed curve marks the spinodal line (the boundary of the thermodynamically unstable region). The diamond symbols with error bars show the solubility limits as obtained by a SGCMC simulation. The shaded region offers a range of possible $\mathrm{Cr}$ solubility limits in Fe as obtained by Xiong et al. [11], while the triangles are results from CE calculations fitted to first principles data [12].

Gibbs [14] showed that the formation free energy $\Delta G_{n}$ of a cluster containing $n$ atoms is in a matrix with composition $c_{m}$ given by:

$\Delta G_{n}=G_{\text {nuc }}\left(T, c_{m}\right) V(n) \rho+F_{i}(T) A(n)$,

where $G_{\text {nuc }}$ is the free energy gain from nucleating an atom of precipitate material, $F_{i}$ is the cost of creating one unit of interface surface, and $V$ and $A$ are the volume and interface area of a particle containing $n$ atoms. The volume and interface terms are in competition. In the thermodynamically meta-stable region of the miscibility gap, the interface term wins over the volume term for all cluster sizes below a critcal size $n$. Above the critical size, the volume term dominates, and the addition of further atoms into the nucleus becomes energetically favorable. Therefore, the critical size is obtained by solving 


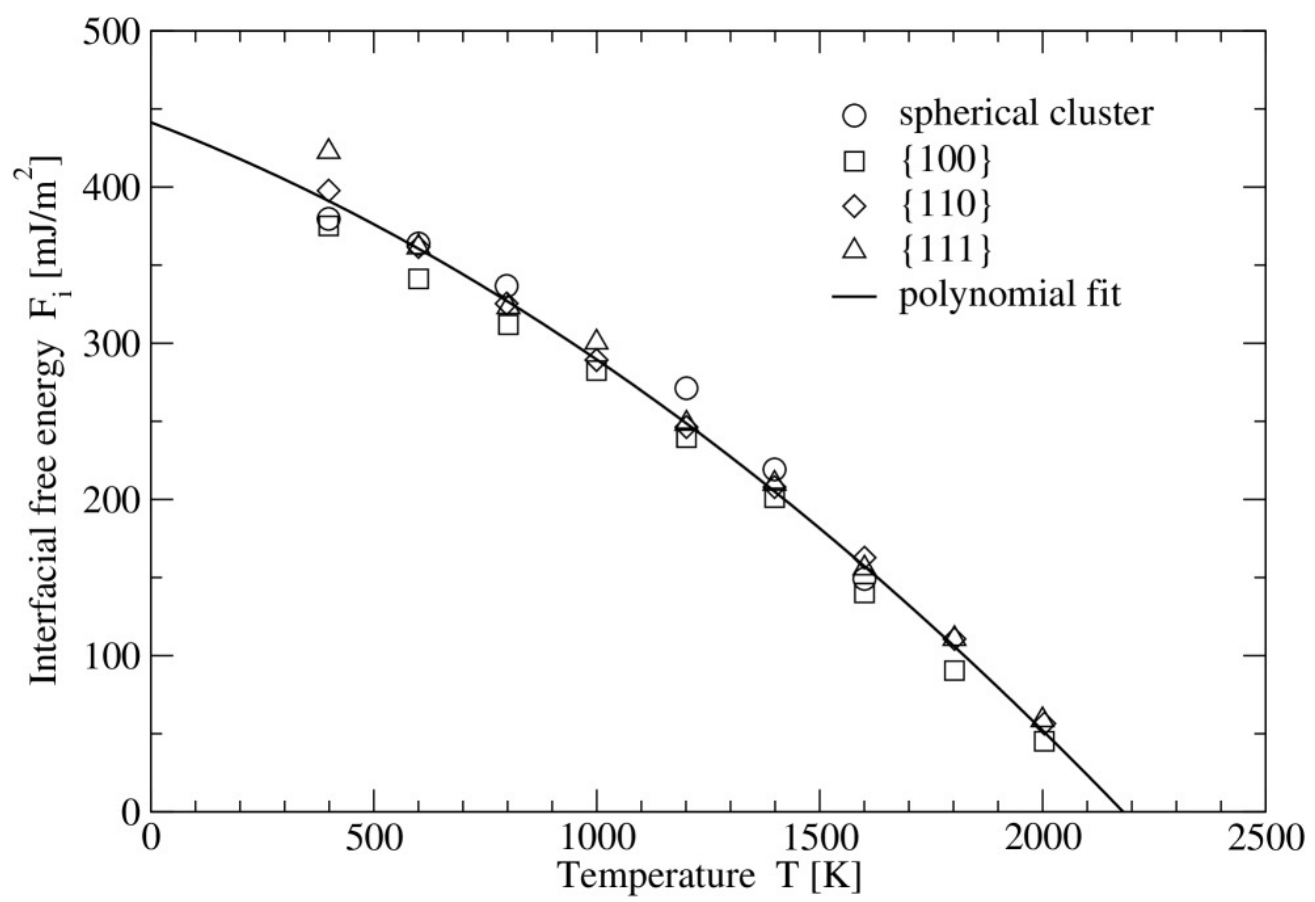

Figure 3. Interfacial free energy of the Fe-Cr system as a function of temperature obtained by Sadigh and Erhart [4] using VDSGCMC simulations. The solid line shows the polynomial fit to the data used in our calculations.

$\left.\frac{d \Delta G_{n}}{d n}\right|_{n^{*}}=0$

In classical nucleation theory, an infinitesimal global change in matrix composition is assumed to balance the creation of a precipitate. However, this can only be valid in the limit of infinite diffusivity. Conservation of mass demands that any fluctuation resulting in a local composition increase must be balanced by an equal solute depletion somewhere in the sample. At low diffusivities over short timescales the depleted region must be in close proximity to the enriched region.

Thus, we consider two limiting cases. The first is an infinitesimal global depletion in solute following classical nucleation theory. The second case is a localized depletion zone around the particle.

For the infinitesimal depletion case we start with the well-known expression for the free energy of nucleation (see Clouet [15] for a comprehensive review): 


$$
G_{n u c}=G\left(c_{\alpha^{\prime}}, T\right)-G\left(c_{m}, T\right)-\left(c_{\alpha^{\prime}}-c_{m}\right) \frac{\partial G}{\partial c}\left(c_{m}, T\right) .
$$

For the second case, we assume that all solute needed to form the nucleus comes from a localized region, which as a zeroth-order approximation can be assumed to consist of a solute-depleted $\alpha$-phase material at a constant equilibrium composition $c_{d}=c_{\alpha}$. We assume this depletion zone to be centered around the particle with a radius $r_{d}$. The volume of the depletion zone $V_{d}(n)$ needed to form a precipitate containing $n$ atoms in a matrix with a solute concentration $c_{m}$ is then given through mass conservation as:

$V_{d}=n \Omega \frac{c_{\alpha^{\prime}}-c_{m}}{c_{m}-c_{\alpha^{\prime}}}$,

where $c_{\alpha}$ and $c_{\alpha}^{\prime}$ are the equilibrium temperature-dependent compositions in the terminal phases. The free energy of nucleation is then given by:

$$
G_{n u с}=G\left(c_{m}, T\right)-G\left(c_{\alpha}, T\right)-\frac{c_{m}-c_{\alpha}}{c_{\alpha^{\prime}}-c_{\alpha}} \cdot\left(G\left(c_{\alpha^{\prime}}, T\right)-G\left(c_{\alpha}, T\right)\right) .
$$

Figure 4 depicts the critical nuclei sizes $n\left(T, c_{m}\right)$ at temperatures $300 \mathrm{~K}, 500 \mathrm{~K}$, and $700 \mathrm{~K}$ for all matrix concentrations $c_{m}$ between the binodal and the spinodal on the Fe-rich side of the phase diagram.

We note that by increasing the local depletion zone composition, thus the depletion zone radius, the classical nucleation theory result can be recovered in the limit of $r_{d} \rightarrow \infty$ and $c_{d} \rightarrow c_{m}$. Both limits then can be considered as representing cases of fast and slow kinetics, respectively.

The thermodynamic nucleation rate has a prefactor that accounts for the shape of the nucleation barrier and the fact that developing critical nuclei can be viewed as performing a random walk in cluster-size space. This prefactor is called the "Zeldovich factor" [16] and is given by:

$$
Z=\sqrt{\left.\frac{-1}{2 \pi k_{B} T} \frac{\partial^{2} G}{\partial n^{2}}\right|_{n^{*}}}
$$

Knowing the entire free energy surface and, consequently, the functional form of the nucleation barrier, this factor is readily computed. Figure 5 shows the results. 


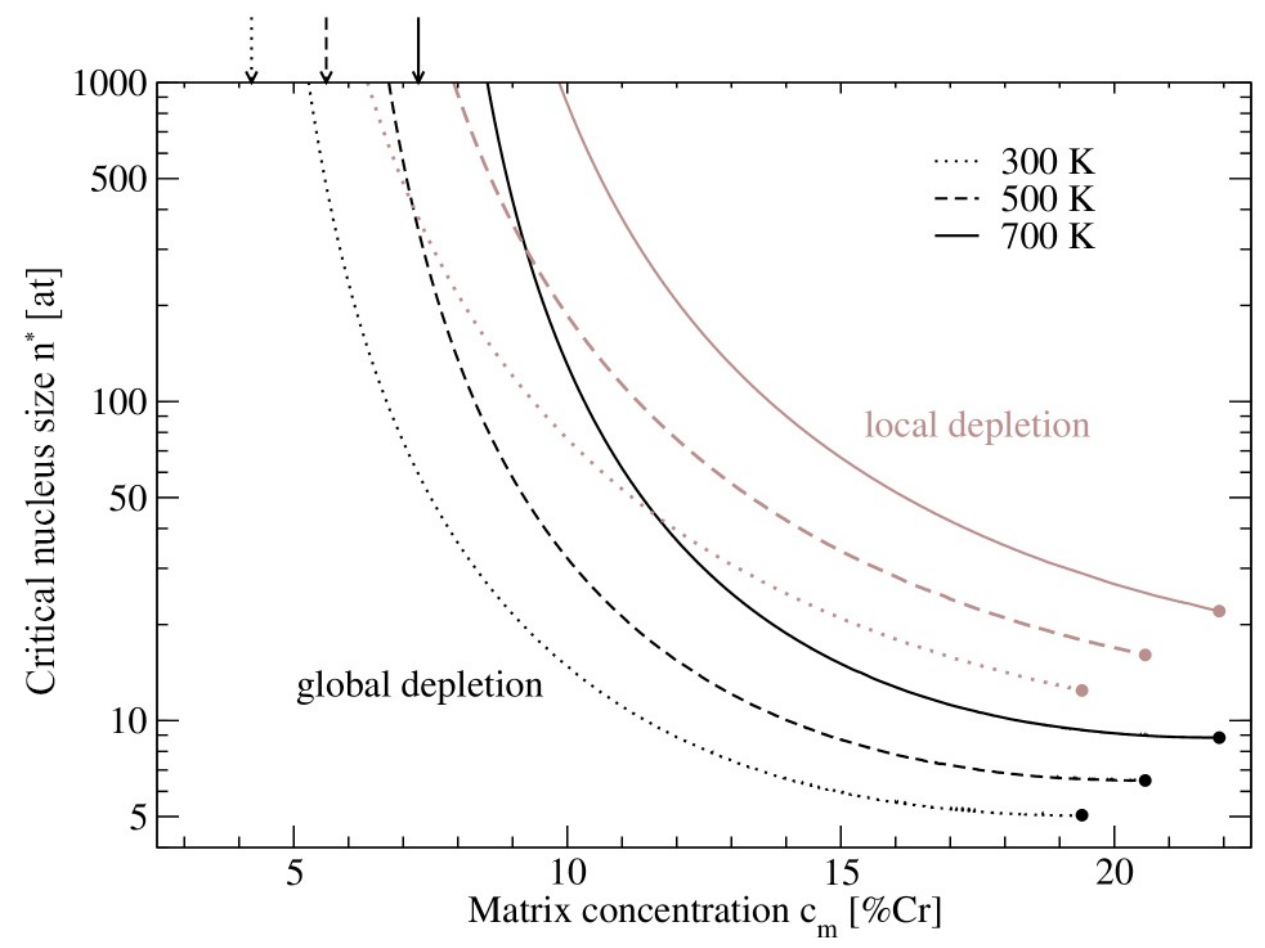

Figure 4. Critical nucleus sizes $\mathrm{n}^{*}$ in number of atoms for the local (gray) and global (black) depletion cases as a function of solute concentration in the matrix at $300 \mathrm{~K}, 500 \mathrm{~K}$, and $700 \mathrm{~K}$. The dotted, dashed, and solid arrows indicate the solubility limit at the three temperatures. The disk symbols indicate the spinodal, beyond which nucleation is replaced by spontaneous phase decomposition.

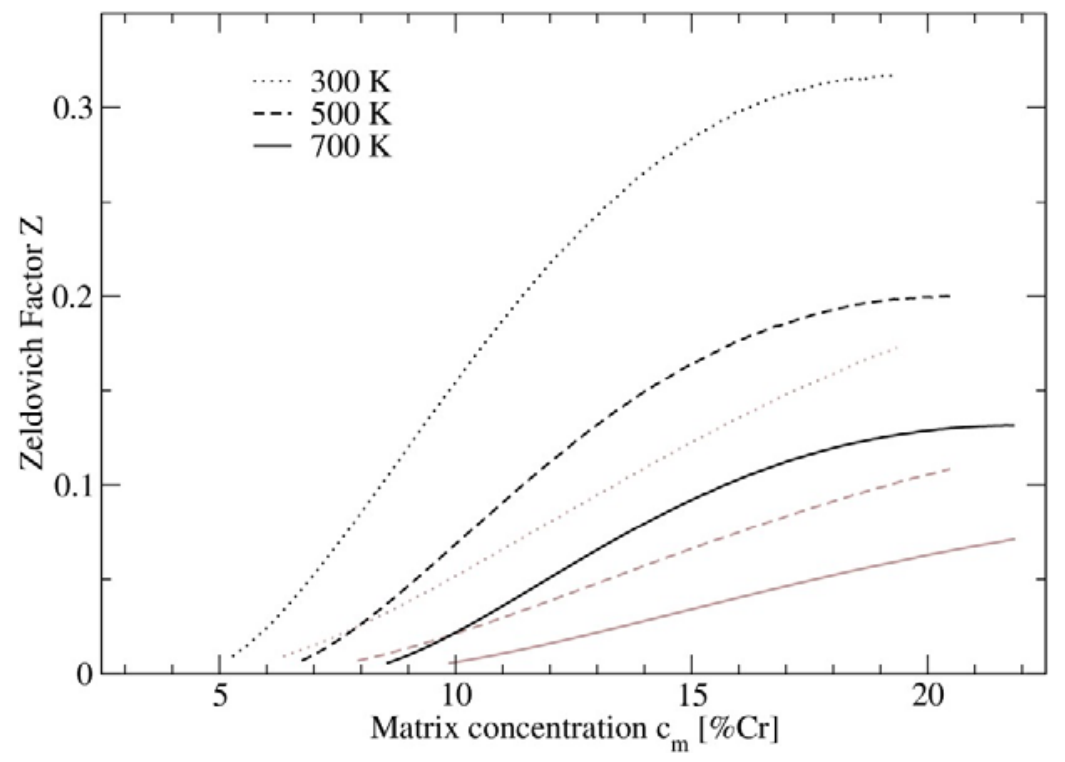

Figure 5. Zeldovich factors $\mathrm{Z}$ for the local (gray) and global (black) depletion cases as a function of solute concentration in the matrix at $300 \mathrm{~K}, 500 \mathrm{~K}$, and $700 \mathrm{~K}$. 
To calculate the actual nucleation rate $R$, the thermodynamic picture has to be complemented by the kinetic side of the problem in the form of a monomer arrival rate $\beta$ determined by the rate-limiting diffusion coefficients. Here, we use the diffusion coefficient of $\mathrm{Cr}$ in $\alpha-\mathrm{Fe}$, given by Lee et al. [17], and calculate the arrival rate as:

$$
\beta^{*}=c_{\text {surf }} \Omega^{\frac{-2}{3}} A \frac{f}{2},
$$

where $f$ is the jump frequency of a solute atom and $c_{\text {surf }} \Omega^{-2 / 3} A$ is the number of solute atoms near the surface of the precipitate. We assume that half of the jumps lead to an addition of a monomer to the particle. The nucleation rate per unit volume is then given by:

$$
R=N \beta^{*} Z e^{\frac{-\Delta G_{n}}{k_{B} T}},
$$

where $N$ is the number of nucleation sites per unit volume, which, in classical nucleation theory, is assumed to be equal to the number of solute monomers. In the global depletion limit we set $c_{\text {surf }}=c_{m}$ and in the local depletion limit we set $c_{\text {surf }}=c_{d}$. Figure 6 illustrates the resulting nucleation rates as a function of solute concentration $c_{m}$.

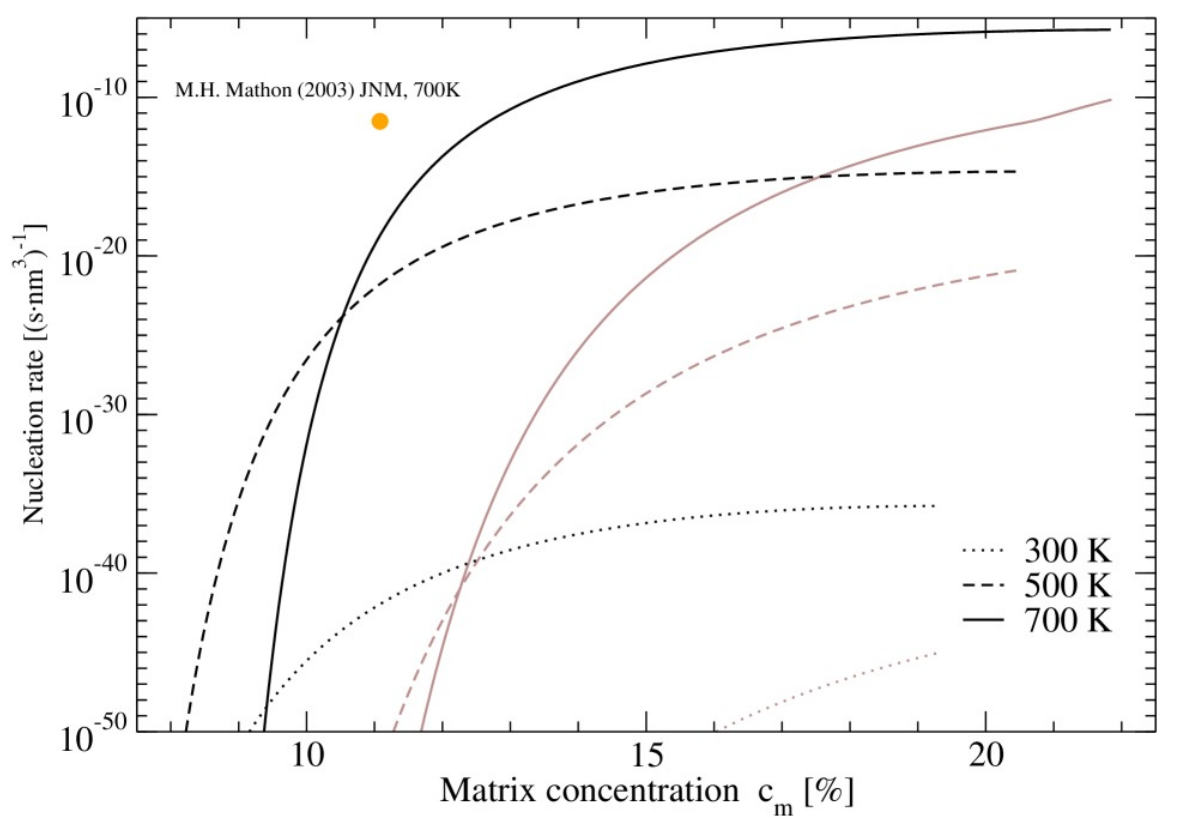

Figure 6. Nucleation rates $\mathrm{R}$ per unit volume for the local (gray) and global (black) depletion cases as a function of solute concentration in the matrix at $300 \mathrm{~K}$, $500 \mathrm{~K}$, and $700 \mathrm{~K}$. A single experimental data point by Mathon et al. [18] on martensitic LA4Ta steel with $11.8 \% \mathrm{Cr}$ at $700 \mathrm{~K}$ is shown in the top left. 
Experimental data for comparison are especially scarce. Data from a single thermal annealing experiment by Mathon et al. [18] on martensitic LA4Ta steel with $11.8 \% \mathrm{Cr}$ at $700 \mathrm{~K}$ is shown in the top left of the plot (Figure 6). Compared to our model alloy, the system in the experiment differs both in morphology and trace element composition. Yet, by taking the size of the result space into account, a reasonable agreement with the simulation is observerd.

The analytical expressions for the critical size and nucleation rate are rather bulky and contain the first and second derivatives of the already complex free energy function. Here, we present simple and computationally inexpensive fitting functions and their parameter sets, to reproduce the critical size $n_{g}$ and nucleation rate $R_{\{, g, g}$ data with reasonable accuracy.

$$
\begin{aligned}
& n_{l}^{*}=\frac{\kappa_{a 1}}{\left(x-\kappa_{a 2}\right)^{6}}+\frac{\kappa_{a 3}}{\left(x-\kappa_{a 2}\right)^{2}} \\
& n_{g}^{*}=\frac{\kappa_{b 1}}{\left(x-\kappa_{b 2}\right)^{\kappa_{b 3}}}+\kappa_{b 4} \\
& R_{\{l, g\}}=\exp \left[\kappa_{\{c, d\} 1}+\kappa_{\{c, d\} 4}\left(x-\kappa_{\{c, d\} 2}\right)^{\kappa_{\{c, d\}\}}}\right] \\
& \kappa_{\{a, b\}^{*}}=\kappa_{\{a, b\}^{*}, 1}+\kappa_{\{a, b\}^{*}, 2} T+\kappa_{\{a, b\}^{*}, 3} T^{2} \\
& \kappa_{\{c, d\}^{*}}=\kappa_{\{c, d\}^{*}, 1}+\kappa_{\{c, d\}^{*}, 2} T+\kappa_{\{c, d\}^{*}, 3} \sqrt{T}
\end{aligned}
$$

The subscripts $l$ and $g$ refer to the local and global depletion cases, respectively. The functions are fit for a temperature range of $300 \mathrm{~K}$ to $700 \mathrm{~K}$. All parameters are listed in Table-2. The fitted critical nucleus sizes match the target within 5\%, and the fitted nucleation rate matches the target within better than one order of magnitude.

This data is useful as input for larger length scale models such as the phase-field method. We give both the critical nucleus size and nucleation rate in terms of computationally efficient fitting functions that can be directly plugged into a coarse-grained simulation code. 


\begin{tabular}{|c|c|c|c|}
\hline & $\kappa * *, 1$ & $\kappa * *, 2$ & $\kappa * *, 3$ \\
\hline$\kappa_{a 1}$ & $4.0563 \cdot 10^{-6}$ & $-5.7096 \cdot 10^{-9}$ & $2.2396 \cdot 10^{-11}$ \\
\hline$\kappa_{a 2}$ & $7.0273 \cdot 10^{-3}$ & $3.8405 \cdot 10^{-5}$ & $3.0734 \cdot 10^{-8}$ \\
\hline$\kappa_{a 3}$ & $3.0597 \cdot 10^{-1}$ & $-2.5131 \cdot 10^{-5}$ & $6.1304 \cdot 10^{-7}$ \\
\hline$\kappa_{b 1}$ & $6.4613 \cdot 10^{-3}$ & $-1.6848 \cdot 10^{-6}$ & $1.7113 \cdot 10^{-8}$ \\
\hline$\kappa_{b 2}$ & $2.7760 \cdot 10^{-2}$ & $4.1084 \cdot 10^{-5}$ & $3.6018 \cdot 10^{-8}$ \\
\hline$\kappa_{b 3}$ & 2.5358 & $-3.0997 \cdot 10^{-5}$ & $-5.0254 \cdot 10^{-8}$ \\
\hline$\kappa_{b 4}$ & 3.3010 & $1.8115 \cdot 10^{-4}$ & $6.6956 \cdot 10^{-6}$ \\
\hline$\kappa_{c 1}$ & -430.6123 & $-4.7526 \cdot 10^{-1}$ & 28.3425 \\
\hline$\kappa_{c 2}$ & $6.3364 \cdot 10^{-2}$ & $1.7403 \cdot 10^{-4}$ & $-4.1923 \cdot 10^{-3}$ \\
\hline$\kappa_{c 3}$ & $-8.9569 \cdot 10^{-1}$ & $2.1468 \cdot 10^{-3}$ & $-9.0857 \cdot 10^{-2}$ \\
\hline$\kappa_{c 4}$ & -3.5363 & $-5.0870 \cdot 10^{-3}$ & $2.5480 \cdot 10^{-1}$ \\
\hline$\kappa_{d 1}$ & -439.0426 & $-5.1439 \cdot 10^{-1}$ & 29.8054 \\
\hline$\kappa_{d 2}$ & $4.2415 \cdot 10^{-2}$ & $1.2897 \cdot 10^{-4}$ & $-2.2891 \cdot 10^{-3}$ \\
\hline$\kappa_{d 3}$ & -2.6509 & $-1.2347 \cdot 10^{-3}$ & $5.6874 \cdot 10^{-2}$ \\
\hline$\kappa_{d 4}$ & $-2.3432 \cdot 10^{-1}$ & $-1.6963 \cdot 10^{-4}$ & $1.1569 \cdot 10^{-2}$ \\
\hline
\end{tabular}

Table-2. Coefficients for the approximate fitting functions for critical nucleus size $\left(\kappa_{\{a, b\}}\right)$ and nucleation rate $\left(\kappa_{\{\mathrm{c}, \mathrm{d}\}}\right)$ (Equations 15-19). 


\section{Phase-field model of homogenous nucleation of $\mathrm{Cr}$ precipitates in $\mathrm{Fe}$-Cr alloys}

In $\mathrm{Fe}-\mathrm{Cr}$ alloys, $\mathrm{Cr}$ precipitate is a $\mathrm{Cr}$-rich phase with the same structure as the matrix phase (bcc Fe-Cr solid solution). Therefore, the precipitate microstructure in bcc Fe- $\mathrm{Cr}$ alloys can be uniqely described by $\mathrm{Cr}$ concentration. In this section, we will introduce the homogenous nucleation mechansim into phase-field formalism using the atomistic data given in the previous section.

In the framework of phase-field approach, the concentration of $\mathrm{Cr}, C_{C r}(\mathbf{r}, t)$, is employed as a phase-field variable, where $\mathbf{r}=\left(r_{1}, r_{2}, r_{3}\right)$ is the spatial coordinate and $t$ is time. Compared with $\mathrm{Cr}$ solubility in $\mathrm{Fe}-\mathrm{Cr}$ alloys, the thermal equilibrium vacancy concentration is very small. Thus, it is ignored in the present model. As such, the concentration of $\mathrm{Fe}$ is $1-C_{C r}$. The total free energy of the binary system can be expressed as:

$F=\int_{V}\left[\frac{N A_{0}}{\Omega_{0}} f\left(C_{C r}, T\right)+\frac{\kappa}{2}\left|\nabla C_{C r}\right|^{2}\right] d V$,

where $T$ is temperature, $\nabla=\left(\partial / \partial r_{1}+\partial / \partial r_{2}+\partial / \partial r_{3}\right)$ is the gradient operator, and $V$ is the volume of the considered system. $N=6.022 \times 10^{23}$ atom $/ \mathrm{mol}$ is the Avogadro's constant. $\Omega_{0}=1.4087 \times 10^{-5} \mathrm{~m}^{3} / \mathrm{mol}$ is the molar volume of bcc Fe, and $A_{0}=1.602 \times 10^{-19} \mathrm{~J} / \mathrm{eV}$. $f\left(C_{C r}, T\right)$ is the free energy density per atom in electron voltage $(\mathrm{eV})$ and is expressed via Eqs. (1)-(5) with the coefficients given in Table-1. Based on these, Figure 7 shows the free energies calculated at given temperatures.

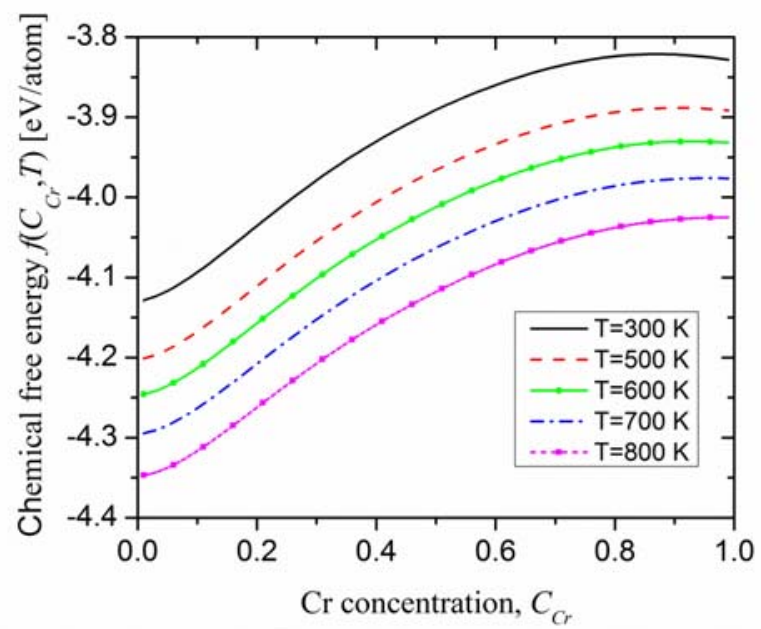

Figure 7. Chemical free energy of Fe-Cr alloys. 
Because concentration $C_{C r}$ is a conserved field variable, its temporal evolution is described by the Cahn-Hilliard equation [19]:

$\frac{\partial C_{C r}}{\partial t}=-\nabla \cdot\left(-M \nabla \frac{\delta F}{\delta C_{C r}}\right)=\nabla \cdot M \nabla\left[\frac{N A_{0}}{\Omega_{0}} \frac{\partial f}{\partial C_{C r}}-\kappa \nabla^{2} C_{C r}\right]$,

where $M$ is the mobility of $\mathrm{Cr}$ and related with its diffusivity, $\mathrm{D}$, as $M=\frac{D \Omega_{0}}{\Re T}$ with $\mathfrak{R}=8.314 \mathrm{~J} /(\mathrm{molK})$ being the gas constant. For numerically solving Eq. (21), the following normalizations are used: $\quad t^{*}=\frac{N A_{0} M}{l_{0}^{2} \Omega_{0}} t, \quad r_{i}^{*}=\frac{r_{i}}{l_{0}}$, $\nabla=\left(\frac{\partial}{\partial r_{1}}, \frac{\partial}{\partial r_{2}}, \frac{\partial}{\partial r_{3}}\right)=\frac{1}{l_{0}}\left(\frac{\partial}{\partial r_{1}^{*}}, \frac{\partial}{\partial r_{2}^{*}}, \frac{\partial}{\partial r_{3}^{*}}\right)=\frac{1}{l_{0}} \nabla^{*}, \quad$ and $\kappa^{*}=\frac{\kappa \Omega_{0}}{N A_{0} l_{0}^{2}}, \quad$ where $\quad l_{0} \quad$ is characteristic length. Then, Eq. (21) is changed into:

$$
\frac{\partial C_{C r}}{\partial t^{*}}=\nabla^{* 2}\left[\frac{\partial f}{\partial C_{C r}}-\kappa^{*} \nabla^{* 2} C_{C r}\right],
$$

To predict Cr precipitate formation and growth, the system's thermodynamic and kinetic properties, including the diffusivity of $\mathrm{Fe}$ and $\mathrm{Cr}$, the interfacial energy, and the nucleation rate of $\mathrm{Cr}$-rich precipitates, are needed.

\section{III.1. Diffusivity of Fe and $\mathrm{Cu}$ in $\mathrm{Fe}$-Cr alloys}

The diffusivity of $\mathrm{Fe}$ in bcc Fe via vacancy mechanism was calculated using the LeClaire model [20], and the values of the vacancy formation energy and migration energy ( $E^{v, f}=2.18 \mathrm{eV}$ and $E^{v, m}=0.65 \mathrm{eV}$, respectively) were obtained from ab initio calculations. The diffusivity of $\mathrm{Fe}$ in the unit of $\mathrm{m}^{2} / \mathrm{s}$ is given by [21]:

$$
D_{b c c_{-} F e}^{F e}=2.051 \times 10^{-5} \exp \left(-\frac{2.84}{k_{B} T}\right)
$$

$\mathrm{Cr}$ diffusivity also can be calculated from the LeClaire model. When including the solute enhancement of solvent diffusion, the Cr diffusion rate was found to be much faster than 
$\mathrm{Fe}$ in both cases of the dilute limit and concentrated $\mathrm{Fe}-10 \% \mathrm{Cr}$ alloys. Kinetic lattice Monte Carlo (KLMC) simulations also showed that Cr diffuses faster (2 150 times) than Fe by a vacancy mechanism [21]. In the present simulations, we assume the diffusivity of $\mathrm{Cr}$ is two orders of magnitude larger than that of Fe (i.e., $D_{b c c_{-} F e}^{C r}=100 D_{b c c_{-} F e}^{F e}$ ). Table3 lists the temperature dependence of $\mathrm{Fe}$ and $\mathrm{Cr}$ diffusivity.

\begin{tabular}{l|l|l} 
Temperature (K) & $D_{b c_{-} F e}^{F e}\left(\mathrm{~m}^{2} / \mathrm{s}\right)$ & $D_{b c c_{-} F e}^{C r}\left(\mathrm{~m}^{2} / \mathrm{s}\right)$ \\
\hline 550 & $1.9 \times 10^{-31}$ & $1.9 \times 10^{-29}$ \\
\hline 600 & $2.8 \times 10^{-29}$ & $2.8 \times 10^{-27}$ \\
\hline 650 & $1.9 \times 10^{-27}$ & $1.9 \times 10^{-25}$ \\
\hline 700 & $7.2 \times 10^{-26}$ & $7.2 \times 10^{-24}$
\end{tabular}

Table-3. Fe and Cr self-diffusivity in Fe-Cr alloys.

\section{III.2. Interfacial energy}

In the phase field model, both the chemical free energy $f\left(C_{C r}, T\right)$ and gradient energy contribute to the interfacial energy. For a given characteristic length $l_{0}$ and gradient coefficient $\kappa$, the interfacial energy of a flat interface can be numerically calculated. To achive this, we put a precipitate at the center of a one-dimensional simulation cell and allowed the system to approach its equilibrium through $\mathrm{Cr}$ diffusion. At the equilibrium state, the concentrations in both the precipitate and matrix reach their equilibrium values, respectively. The equilibrium interface concentration profile is obtained correspondingly. The interfacial energy is numerically calculated with the equilibrium concentration profile by:

$\gamma=\frac{N A_{0}}{2 \Omega_{0}} \int_{V}\left[f\left(C_{C r}, T\right)+\frac{\kappa^{*}}{2}\left|\nabla C_{C r}\right|^{2}\right] d V=\frac{N A_{0}}{2 \Omega_{0}} \int_{-\infty}^{\infty}\left[f\left(C_{C r}, T\right)+\frac{\kappa^{*}}{2}\left|\nabla C_{C r}\right|^{2}\right] d r_{1}$.

If we define the interface to be the region that the $\mathrm{Cr}$ concentration falls within $0.05 C_{C r}^{P, e q}$ 
and $0.95 C_{C r}^{P, e q}$, we can calculate the interface thickness from the equilibrium interfacial concentration profile, where $C_{C r}^{P, e q}$ is the $\mathrm{Cr}$ equilibrium concentration in the precipitate phase. Thus, through tailoring the characteristic length $l_{0}$ and gradient coefficient $\kappa$, the phase field model can correctly describe the interfacial properties, i.e., interfacial thickness and interfacial energy of $\mathrm{Cr}$ precipitates.

\section{III.3. Nucleation rate and nucleation scheme}

In the classical nucleation theory [22-25], for simplicity, it is assumed the concentration in the nucleus is more or less constant throughout its volume. Its interface is sharp, while the interaction among nuclei is ignored and nucleation barrier $\Delta G^{*}$, critical size $R^{*}$, incubation time $\tau$, atomic impingement rate $\beta^{*}$ remain unchanged. Under these assumptions, the nucleation rate can be calculated by:

$J^{*}(t)=Z \beta^{*} N_{0} \exp \left(-\Delta G^{*} / k_{B} T\right) \exp (-\tau / t)$

where $N_{0}$ is lattice sites per unit volume of the crystal and $Z$ is the Zeldovich factor. The assumptions in the classical nucleation theory can be partially released in the nonclassical nucleation theory developed by Cahn and Hilliard [19,26]. The phase-field method describes bulk energy, interfacial energy, long-range interaction energy, and microstructures in the same way as the non-classical nucleation theory. It has been successfully used to predict thermodynamic properties of critical nuclei, including critical sizes, critical shapes, nucleation barriers, and critical composition fluctuations [26,27]. However, modeling the nucleation process is one of the great challenges in phase-field simulations. Here, we propose a general nucleation scheme to introduce the critical nuclei into the simulation cell. The scheme is similar to the nucleation process proposed in [28] but it is more general. It is based on the nucleation probability calculated from the nucleation rate in the classical and/or non-classical nucleation theory. According to the nucleation theory, the probability of forming a nucleus at one atomic site in one characteristic nucleation time interval can be calculated by Eq. (24). We consider the 
nucleation rate in a small representative volume $V_{M_{1}}$, consisting of $M_{1}$ phase-field grids during $\Delta t_{M}$ time interval. Usually, $\Delta t_{M}$ is much larger than the incubation time $\tau$. Thus, the total number of nuclei formed in the representative volume $V_{M_{1}}$ during $\Delta t_{M}$ is

$M_{0}=J^{*} \Delta t_{M}=Z N_{0} M_{1} \beta^{*} e^{-\Delta G^{*} / k_{B} T} \Delta t_{M}$.

In the nucleation scheme, we assume that:

1) Transition driving force $\Delta G_{a}$ is proportional to local super-saturation $\Delta C=C_{C r}-C_{C r}^{M, e q}(T) . \quad$ For a two-dimensional model, we have $\Delta G^{*}=\pi \gamma^{2} / \Delta G_{a}=\pi \gamma^{2} /($ const $\Delta C)$ and $M_{0}=J^{*} \Delta t_{M}=\kappa_{1} e^{-\kappa_{2} / \Delta C} \Delta t_{M}$, where $\kappa_{1}=Z N_{0} M_{1} \beta^{*}$ and $\kappa_{2}=\pi \gamma^{2} /\left(\right.$ const $\left._{B} T\right)$.

2) The nucleation may take place at the point with the maximum supersaturation in the representative volume $V_{M_{1}}$ with the probability of $P=1-\exp \left(-M_{0}\right)$.

3) The probability of nucleation $P$ is zero once a $\mathrm{Cr}$ precipitate in $V_{M_{1}}$ already exists and/or the total amount of $\mathrm{Cr}$ in $V_{M_{1}}$ is less than that required to form a critical nucleus.

In the simulations, we use the following scheme to generate the nucleus in the simulation cell. At each nucleation time:

1) randomly search for a small representative volume $V_{M_{1}}$;

2) calculate the nucleation probability at the point with the maximum supersaturation in $V_{M_{1}}$;

3) determine whether or note a nucleus, which has critical concentration profile, should be added at the nucleation site;

4) repeat steps 1 through 3 until the whole simulation cell has been searched.

\section{III.4. Results and discussions}

The model parameters $l_{0}=0.43 \mathrm{~nm}, \kappa^{*}=0.03, d t^{*}=0.007, \kappa_{1}=0.006, \kappa_{2}=0.6$, and $\Delta t_{M}=1.76 t^{*}$ are used in the following simulations. 


\section{III.4.1 Interfacial energy}

First, we numerically calculate the interfacial energy of a flat interface at different temperatures of $T=300 \mathrm{~K}, 400 \mathrm{~K}, 500 \mathrm{~K}, 600 \mathrm{~K}$ and $700 \mathrm{~K}$, respectively. The simulation is carried out in a one-dimensional simulation cell of $1024 d r \times 1 d r \times 1 d r$, including a Cr-rich precipitate with the radius of $R_{0}=5 d r$ at the center of the simulation center. Inside the Cr-rich precipitate, the initial concentration is $C_{C r}=C_{C r}^{P, e q}$ and $C_{C r}=C_{0}=0.13$ for the rest of the simulation cell. Periodic boundaries are applied in $r_{1^{-}}, r_{2}-$, and $r_{3^{-}}$- directions. For each temperature, the equilibrium profile of $C_{C r}$ can be reached by solving Eq. (22). Figure 8a plots the $\mathrm{Cr}$ concentration temporal evolution at $T=600 \mathrm{~K}$ in half of the simulation cell. The origin is the center of the precipitate.
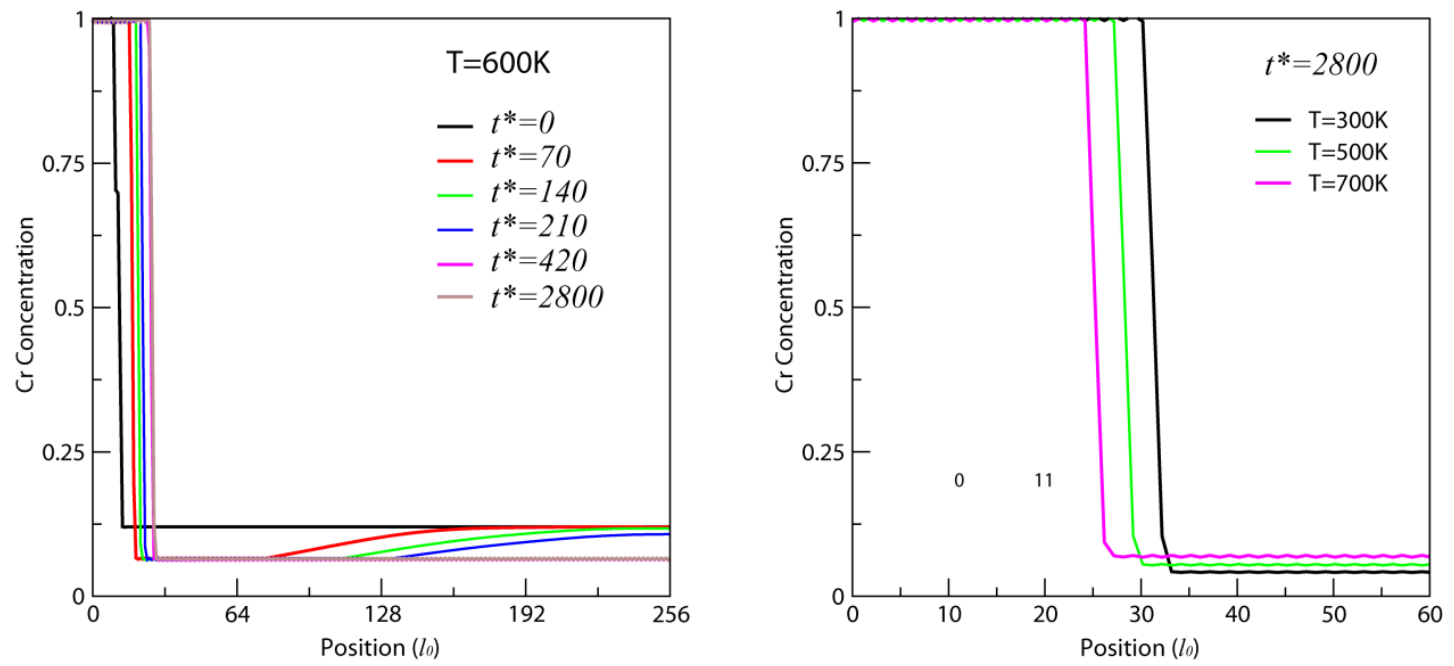

Figure 8. (a) Temporal evolution of $\mathrm{Cr}$ concentration when the initial $\mathrm{Cr}$ concentration is $C_{0}=0.13$ and (b) the equilibrium $\mathrm{Cr}$ concentration profiles for $\mathrm{T}=300 \mathrm{~K}, 500 \mathrm{~K}$ and $700 \mathrm{~K}$, respectively.

Clearly, while the Cr precipitate grows, the concentrations in both the precipitate and matrix approach their equilibrium values $C_{C r}^{P, e q}$ and $C_{C r}^{M, e q}$, respectively. Figure $8 \mathrm{~b}$ depicts the equilibrium $\mathrm{Cr}$ concentration profiles for $T=300 \mathrm{~K}, 500 \mathrm{~K}$ and $700 \mathrm{~K}$. Using the equilibrium $\mathrm{Cr}$ concentration profiles, the interfacial energy can be calculated with Eq. (23). Table-4 lists the interfacial energies of the flat interface calculated from the phase-field model. For comparison, the results from atomistic simulations are also listed. We can see the interface energies from atomistic and phase field simulations have similar 
temperature dependency, i.e., decrease with increasing temperature. However, the interfacial energy predicted from the phase field model is slightly higher than that from the atomistic simulations.

\begin{tabular}{|l|l|l|}
\hline $\begin{array}{l}\text { Temperature } \\
(\mathrm{K})\end{array}$ & $\begin{array}{l}\text { Phase-field } \\
\left(\mathrm{J} / \mathrm{m}^{2}\right)\end{array}$ & $\begin{array}{l}\text { Atomic calculation } \\
\left(\mathrm{J} / \mathrm{m}^{2}\right)\end{array}$ \\
\hline 300 & 0.58 & 0.405 \\
\hline 400 & 0.56 & 0.391 \\
\hline 500 & 0.53 & 0.376 \\
\hline 600 & 0.50 & 0.361 \\
\hline 700 & 0.49 & 0.344 \\
\hline
\end{tabular}

Table-4 Interfacial energies from phase-field simulations

\section{III.4.2 Critical size of a nucleus in a three-dimensional model}

Critical nucleus sizes are examined in a three-dimensional model. The simulations are performed in a simulation cell $128 d r \times 128 d r \times 128 d r$ with periodic boundary conditions in $r_{1^{-}}, r_{2}-$, and $r_{3^{-}}$- directions. The simulation starts with uniform $\mathrm{Cr}$ concentrations of $C_{C r}=0.11,0.13,0.15,0.17$, and 0.19 , respectively. The temperature is set at $600 \mathrm{~K}$. During simulations, $\mathrm{Cr}$ nuclei are introduced continuously into the simulation cell with the previously described nucleation scheme. For a given concentration, nuclei with different sizes are introduced in different runs. The added nucleus shrinks when its size is smaller than the critical size, but it grows when its size is larger than the critical size. Figure 9 summarizes the evolving microstructure from the simulations, and Table-5 presents the predicted critical nucleus sizes from both the phase field modeling and atomistic calculations described earlier. The results show the predicted critical nucleus size with the phase field model is in good agreement with the the atomistic results of the local depletion mechanism. Similar to the molecular dynamics simulations, the current model also uses the local depletion mechanism when introducing a nuclei. 


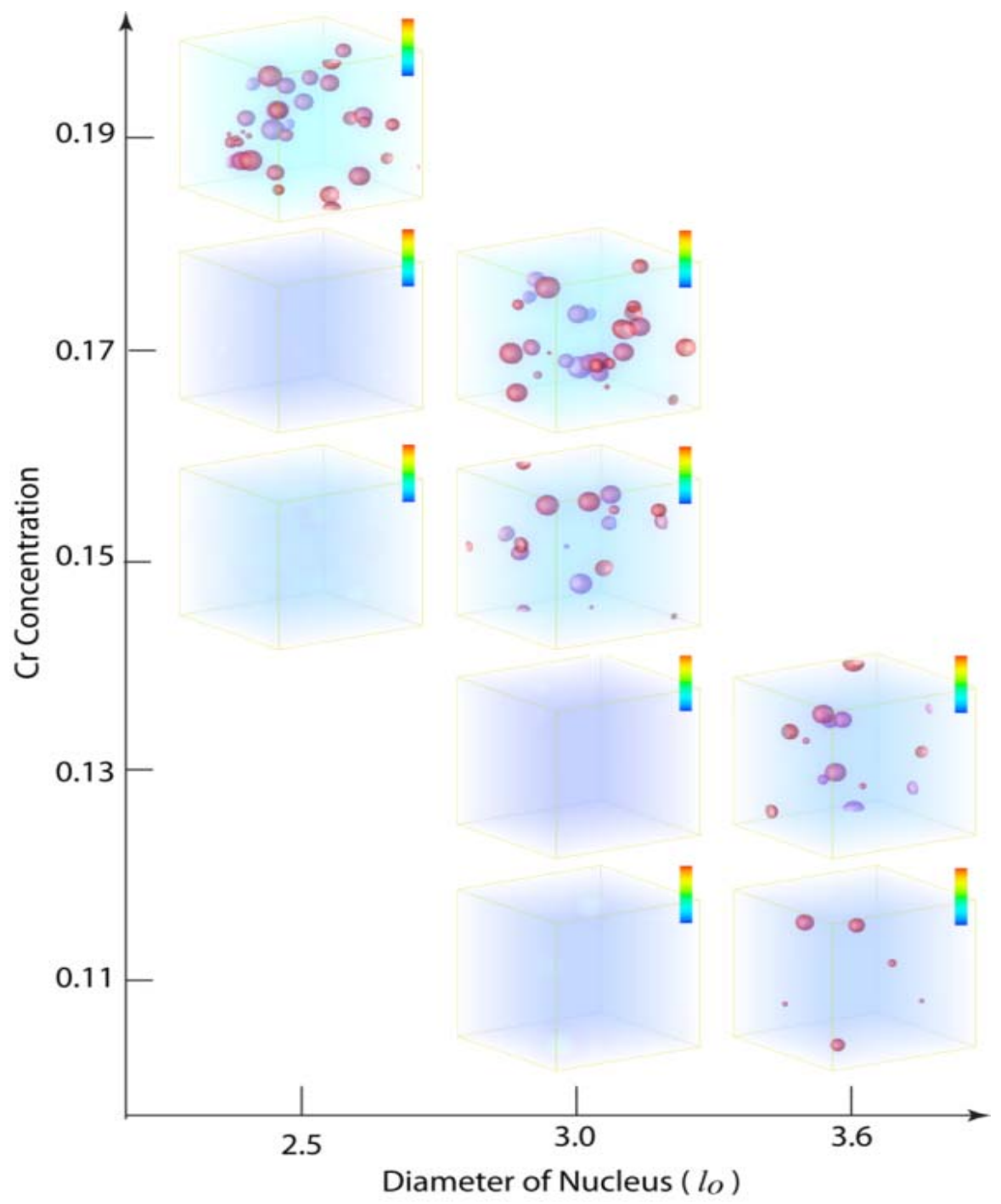

Figure 9. Dependence of the microstructure on the nucleus size and initial $\mathrm{Cr}$ concentration. The red isosurface denotes the interface of $\mathrm{Cr}$ precipitates with $C_{C r}=0.5$. The color bar shows $\mathrm{Cr}$ concentration from 0 to 1.0 .

\begin{tabular}{|l|l|l|l|l|l|}
\hline $\begin{array}{l}\text { Cr } \\
\text { concentration }\end{array}$ & $\begin{array}{l}\text { Diameter } \\
\text { (phase field } \\
\text { model, } \\
l_{0}=0.43 \mathrm{~nm} \text { ) }\end{array}$ & $\begin{array}{l}\text { No. of atoms } \\
\text { (Global } \\
\text { depletion) }\end{array}$ & $\begin{array}{l}\text { Diameter } \\
\text { (Global } \\
\text { depletion) }\end{array}$ & $\begin{array}{l}\text { No. of atoms } \\
\text { (Local } \\
\text { depletion) }\end{array}$ & $\begin{array}{l}\text { Diameter } \\
\text { (Local } \\
\text { depletion) }\end{array}$ \\
\hline 0.11 & $4.0 l_{0}$ & 33 & $2.65 l_{0}$ & 189 & $4.74 l_{0}$ \\
\hline 0.13 & $4.0 l_{0}$ & 17 & $2.12 l_{0}$ & 82 & $3.59 l_{0}$ \\
\hline 0.15 & $3.0 l_{0}$ & 11 & $1.84 l_{0}$ & 48 & $3.00 l_{0}$ \\
\hline 0.17 & $3.0 l_{0}$ & 9 & $1.72 l_{0}$ & 33 & $2.65 l_{0}$ \\
\hline 0.19 & $<2.5 l_{0}$ & 8 & $1.65 l_{0}$ & 24 & $2.38 l_{0}$ \\
\hline
\end{tabular}

Table-5. Critical size (spherical diameter) of $\mathrm{Cr}$ precipitates in $\mathrm{Fe}-\mathrm{Cr}$ alloys at $\mathrm{T}=600 \mathrm{~K}$. 


\section{III.4.3. Composition dependence of nucleation rate in a two-dimensional model}

Nucleation and growth of $\mathrm{Cr}$ precipitates are simulated in a two-dimensional simulation cell of $512 d r \times 512 d r \times 1 d r$ for different initial $\mathrm{Cr}$ concentrations at $T=600 \mathrm{~K}$. In the simulations, nuclei with the critical sizes listed in Table-5 are introduced continuously into the simulation cell with the nucleation scheme (previously described). Figure 10 presents the time evolution of the $\mathrm{Cr}$ precipitates. In the figure, the color bar describes the $\mathrm{Cr}$ concentrations, and the red particles are $\mathrm{Cr}$ precipitates. Figure 11 shows the evolution of total numbers of the survived nuclei and average $\mathrm{Cr}$ concentration in the simulation cell. The temporal evolution of nucleation rates are plotted in Figure 12. From the results in Figures 10-12, it is evident that:

1) Nucleation occurs only at the early stage. The lower the initial $\mathrm{Cr}$ concentration, the longer the nucleation period.

2) The nucleation rate, which is the first derivative of the total nucleus number with respect to time, increases with the increase of the initial $\mathrm{Cr}$ concentration.

3) The total $\mathrm{Cr}$ concentration is conserved during nucleation and growth, which can be seen in Figure 11b.
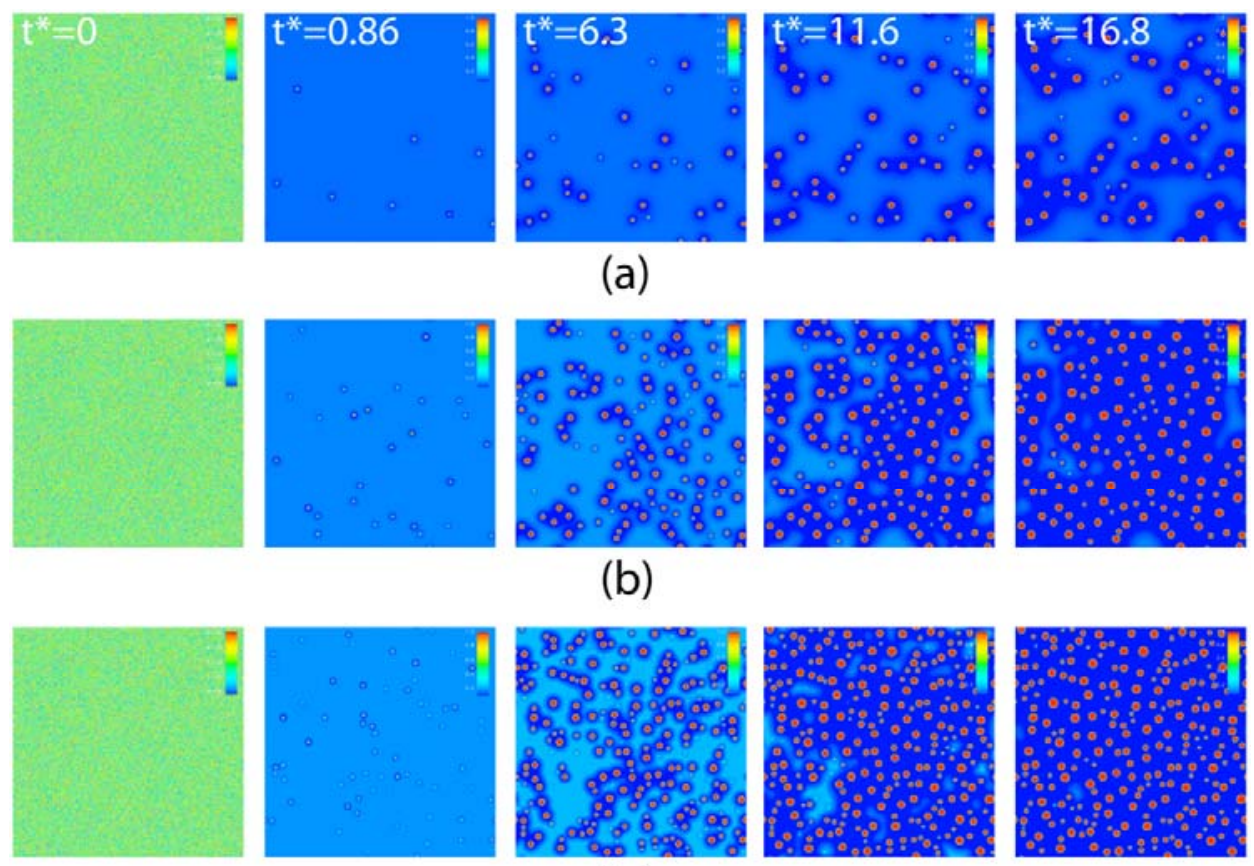

(a)
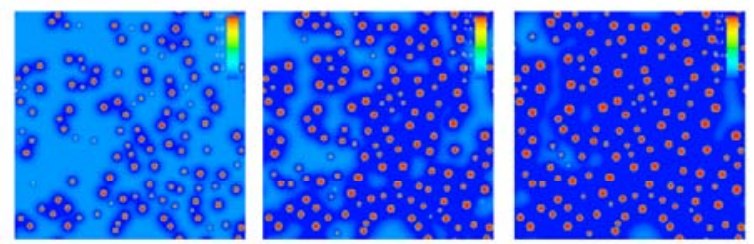

(b)
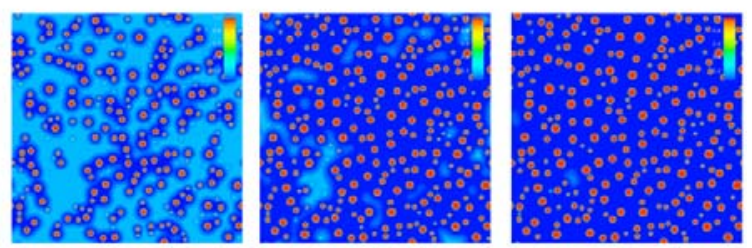

(c)

\section{Time}

Figure 10. Two-dimensional temporal evolution of $\mathrm{Cr}$-rich precipitates via nucleation and growth: (a) $C_{C r}=C_{0}=0.11$, (b) $C_{C r}=C_{0}=0.15$, and (c) $C_{C r}=C_{0}=0.19$. 

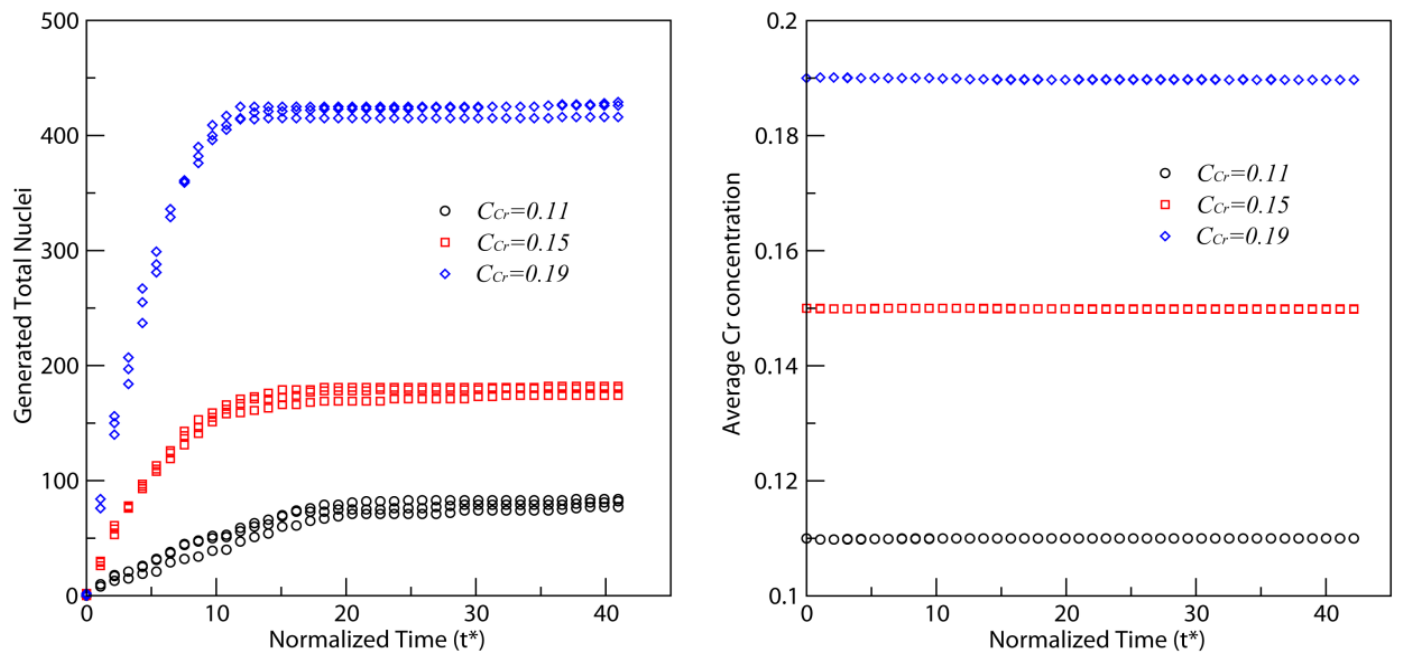

Figure 11. (a) Total nucleus number versus time and (b) average Cr concentration versus time.

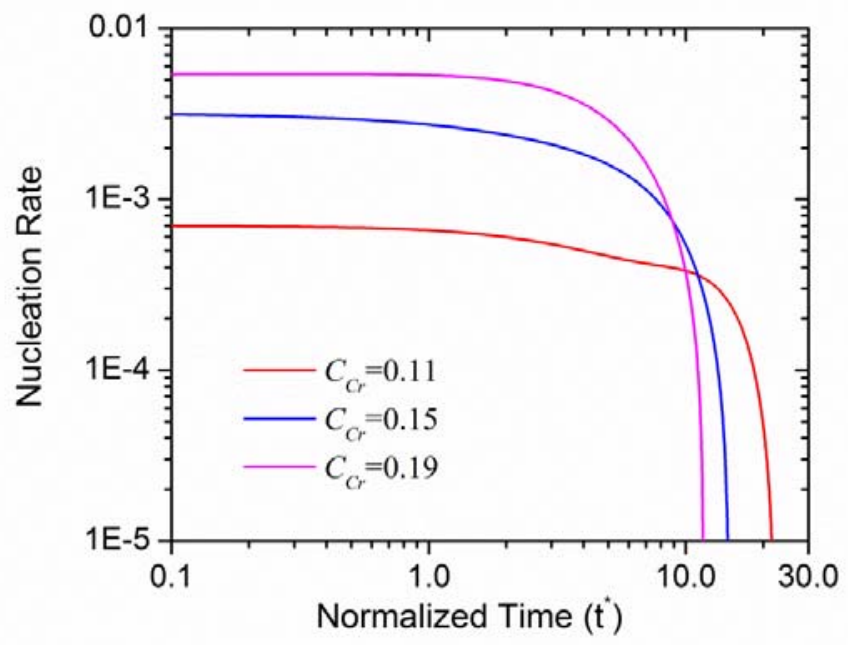

Figure 12. Time dependence of nucleation rate for different initial $\mathrm{Cr}$ concentration at $\mathrm{T}=600 \mathrm{~K}$.

For the case with the initial $\mathrm{Cr}$ concentration of $C_{C r}=\mathrm{C}_{0}=0.17$ and $\mathrm{T}=600 \mathrm{~K}$, the whole aging process from nucleation and growth to coarsening is simulated. Figure 13 shows the plot of $\left(R / l_{0}\right)^{3}$ versus $t^{*}$. A linear relationship between $\left(R / l_{0}\right)^{3}$ and $t^{*}$ holds in the coarsening process and is in agreement with the Lifshitz-Slyozov-Wagner (LSW) theory of Ostwald ripening [30,31]. 


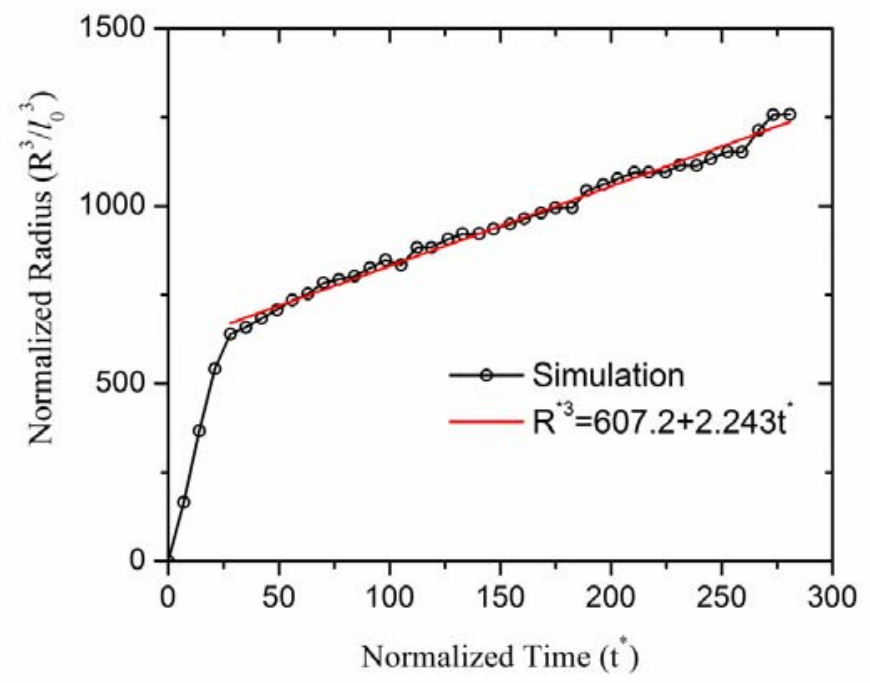

Figure 13. The predicted relationship between mean radius $R$ and time during coarsening for the case with initial $\mathrm{Cr}$ concentration of 0.17 at $T=600 \mathrm{~K}$.

\section{Heteregenous nucleation behavior due to the presence of dislocation in $\mathrm{Fe}-\mathrm{Cr}$ systems}

It is now well established that the presence of sub-microstructural features, such as dislocations and grain boundaries, provides thermodynamically favorable sites for heteregenous nucleation and alters the kinetics of the evolution by providing different diffusional pathways. In this section we model the precipitation behavior due to the presence of dislocations in the system.

The total free energy of the system given in Eq. 20 can be expended to include other energy terms arising from other internal and external driving forces, such as the stored elastic energy $E_{e l}$ in the system:

$$
F=\int_{V}\left[\frac{N A_{0}}{\Omega_{0}} f\left(C_{C r}, T\right)+\frac{\kappa}{2}\left|\nabla C_{C r}\right|^{2}+E_{e l}\right] d V
$$

The elastic strain induced to the system by the composition inhomogeneity is the difference between the total strain $\varepsilon_{i j}$ and the eigenstrain $\varepsilon_{i j}^{0}$, which is taken as: 
$\varepsilon_{i j}^{o}=\varepsilon_{0} \delta_{i j} c$

where $\delta_{i j}$ is the Kronecker-delta function and $\varepsilon_{o}$ is the lattice expansion coefficient which is $\varepsilon_{0}=\left(\frac{1}{a}\right)\left(\frac{d_{F e}}{d_{C r}}\right)$ and taken as 0.006 in this study. Hooke's law links the stress tensor to the strain tensor, and in the presence of eigenstrain, takes the form of:

$\sigma_{i j}=\operatorname{Cijkl}\left(\varepsilon_{k l}-\varepsilon_{k l}^{0}\right)$

The elastic constants were used in the construction of the elasticity tensor, $C_{i j k l}$, which is given in Table-6 [32]. Vegard's law was used to calculate the position-dependent elastic constants using the values from Table-6.

\begin{tabular}{|l|l|l|}
\hline & $\mathrm{Fe}$ & $\mathrm{Cr}$ \\
\hline $\mathrm{C}_{11}$ & 233.10 & 350.00 \\
\hline $\mathrm{C}_{12}$ & 135.44 & 67.80 \\
\hline $\mathrm{C}_{44}$ & 178.30 & 100.08 \\
\hline
\end{tabular}

Table-6. Elastic constants of $\mathrm{Fe}$ and $\mathrm{Cr}$ used in the simulations. The units are in GPa.

The dislocation stress-strain fields can be introduced into the phase-field formalism in a variety of ways [33]. In this study, we adopted the eigenstrain formalism of an edge dislocation given by Mura [34]. For a straight edge dislocation, the resulting eigenstrain has only one component and it can be described by:

$\varepsilon_{21}^{D}=\frac{1}{2} b \delta\left(x_{2}\right) H\left(-x_{1}\right)$

where $b$ is the magnitude of the Burgers vector, $\delta\left(x_{2}\right)$ is the Dirac delta function, and $H\left(-x_{1}\right)$ is the Heaviside step function for a local coordinate system $\left(x_{1}, x_{2}\right)$ located at 
the dislocation. With this implementation into our phase-field formalism, the resulting stress fields for an edge dislocation dipole are shown in Figure 14. As seen from the figure, the well-known stress fields are quite accurately reproduced even with a coarsegrid simulation.
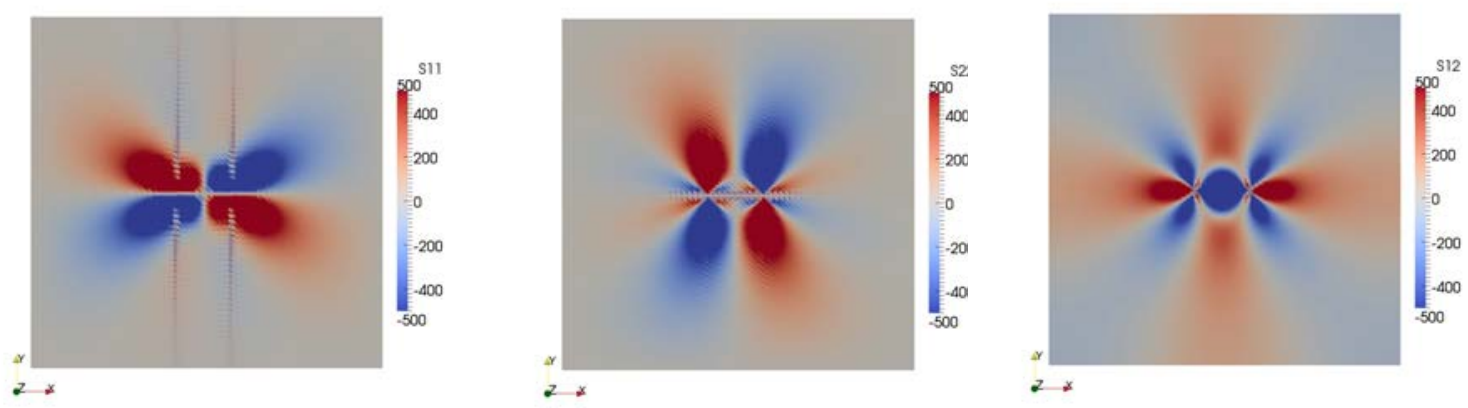

Figure 14. The stress fields of an edge dislocation dipole obtained with eigenstrain formalism.

Then, the elastic strain energy density per unit volume can be obtained from:

$E_{e l}(x, t)=\frac{1}{2} C_{i j k l}\left(\varepsilon_{i j}-\varepsilon_{i j}^{0}+\varepsilon_{i j}^{D}\right)\left(\varepsilon_{k l}-\varepsilon_{k l}^{0}+\varepsilon_{k l}^{D}\right)$,

while the evolution equation takes the form of:

$\left(\frac{\partial c(x, t)}{\partial t}\right)=M \nabla^{2}\left[\frac{\delta f}{\delta c}-\kappa \nabla^{2} c+\frac{\delta E_{e l}}{\delta c}\right]$.

Because we assumed constant mobility $M$, Eq. 30 can be efficiently solved via the spectral method [35]. In addition, the following nondimensional form of Eq. 30 was adopted in the solution:

$x^{*}=x / b, \quad t^{*}=t M \mu V_{m} / b^{2}, \quad f^{*}=f / \mu, \quad \kappa=\kappa /\left(\mu b^{2}\right), \quad E_{e l}=E_{e l} / \mu$

in which $\mu$ is the shear modulus of $\mathrm{Cr}$ (Table-6) and $b$ is the magnitude of the Burgers vector, taken as $10^{-10} \mathrm{~m}$. 


\section{IV.1. Results and discussion}

In the first set of simulations, the aging of $20 \mathrm{at} . \% \mathrm{Cr}$ alloy at $535 \mathrm{~K}$ is considered. In these simulations, a composition fluctuation of \pm 0.0025 with a random distribution is introduced into the system at the first time step to facilitate the phase separation. The spatial and temporal evolution of the aging process is shown in Figure 15.

As shown in the figure, although a relatively large compositional fluctuation was introduced into the system, the nucleation of the $\mathrm{Cr}$ precipitates takes quite some time (between 2000 and 4000 reduced time units). At a reduced time of 4000, the formation of stable Cr precipitates is apparent. Both the nucleation and coarsening of the earlynucleated precipitates continues to the reduced time of 6000 . Thereafter, the coarsening of the large precipitates at the expense of smaller ones with Oswald ripening can be clearly inferred from the figure.

In the next set of simulations, an edge dislocation dipole (composed of a positive and a negative edge dislocation, 50 nondimensional units apart from each other) was introduced into the system. Figure 16 summarizes the predicted spatial and temporal evolution of the aging process in this case. As shown in the figure, the results clearly illustrate a much earlier nucleation of two stable precipitates at the tensile regions of hydrostatic stress in the presence of dislocations at the reduced time step of 2000. Although the overall evolution proceeds similar to the cases described earlier, there are differences in the case with dislocations. As seen at the reduce time of 4000, there are only a few nuclei of precipitates near the dislocations, due to the formation of the denuded zones resulting from the local depletion of the $\mathrm{Cr}$ concentration. In addition, these two early nucleated precipitates at the dislocations continuously grow in size and became the largest ones compared to any of the other precipitates formed later during the course of the simulation. 

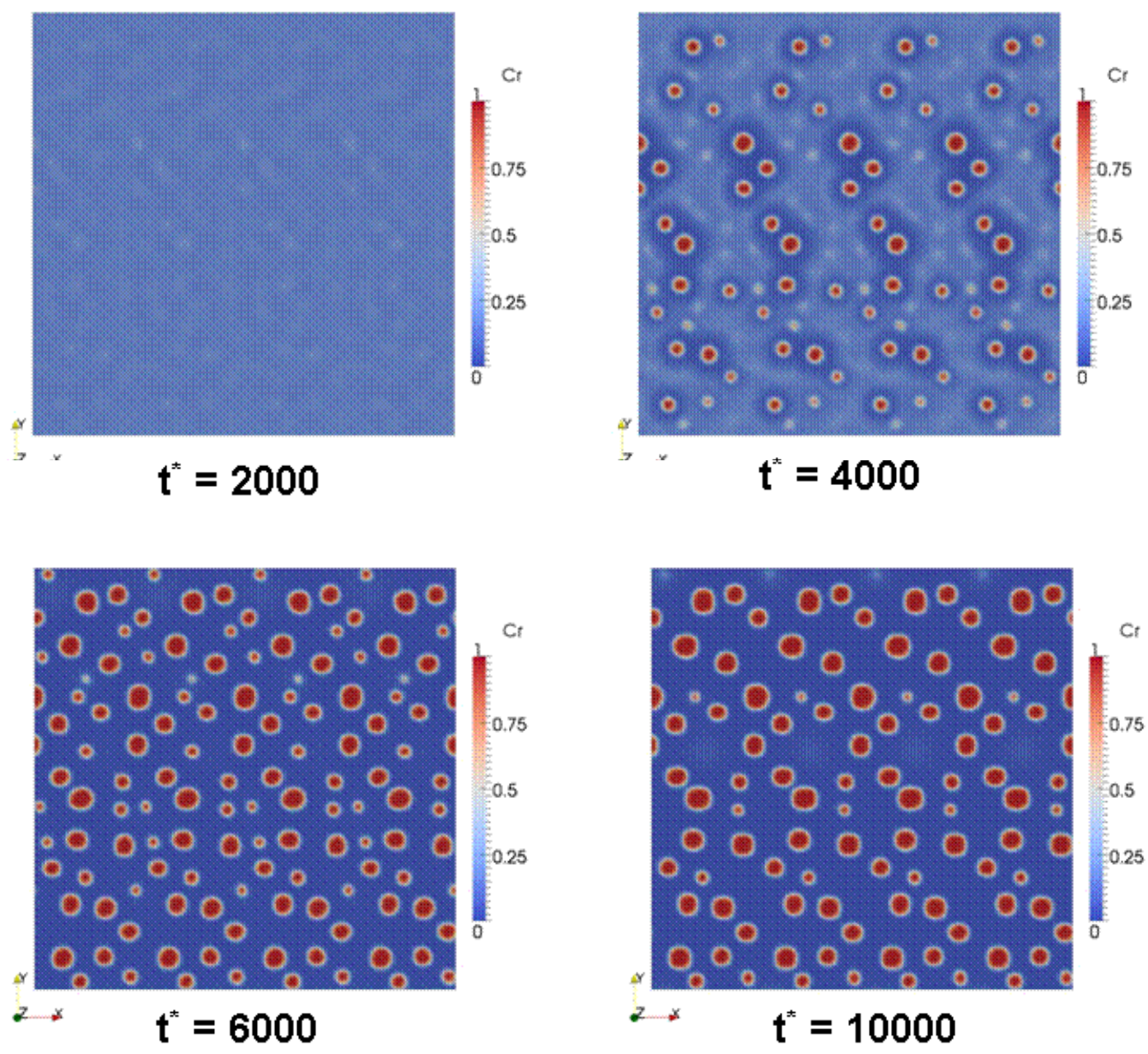

Figure 15. Microstructural evolution behavior of $\mathrm{Fe}-\mathrm{Cr}$ alloy with $20 \mathrm{at} . \% \mathrm{Cr}$ at $535 \mathrm{~K}$.

The microstructural evolutions described above can be more effectively quantified as shown in Figure 17. In this figure, the time evolution of the concentration gradient along a line going through the simulation cell located midway along the $x_{2}$ direction is shown for both cases. In the figure, in addition to the magnitude of the local fluctuations of the composition, the value of the highest peak gives the local concentration of the precipitates, while changes in the peaks' widths indicate the coarsening rates. 

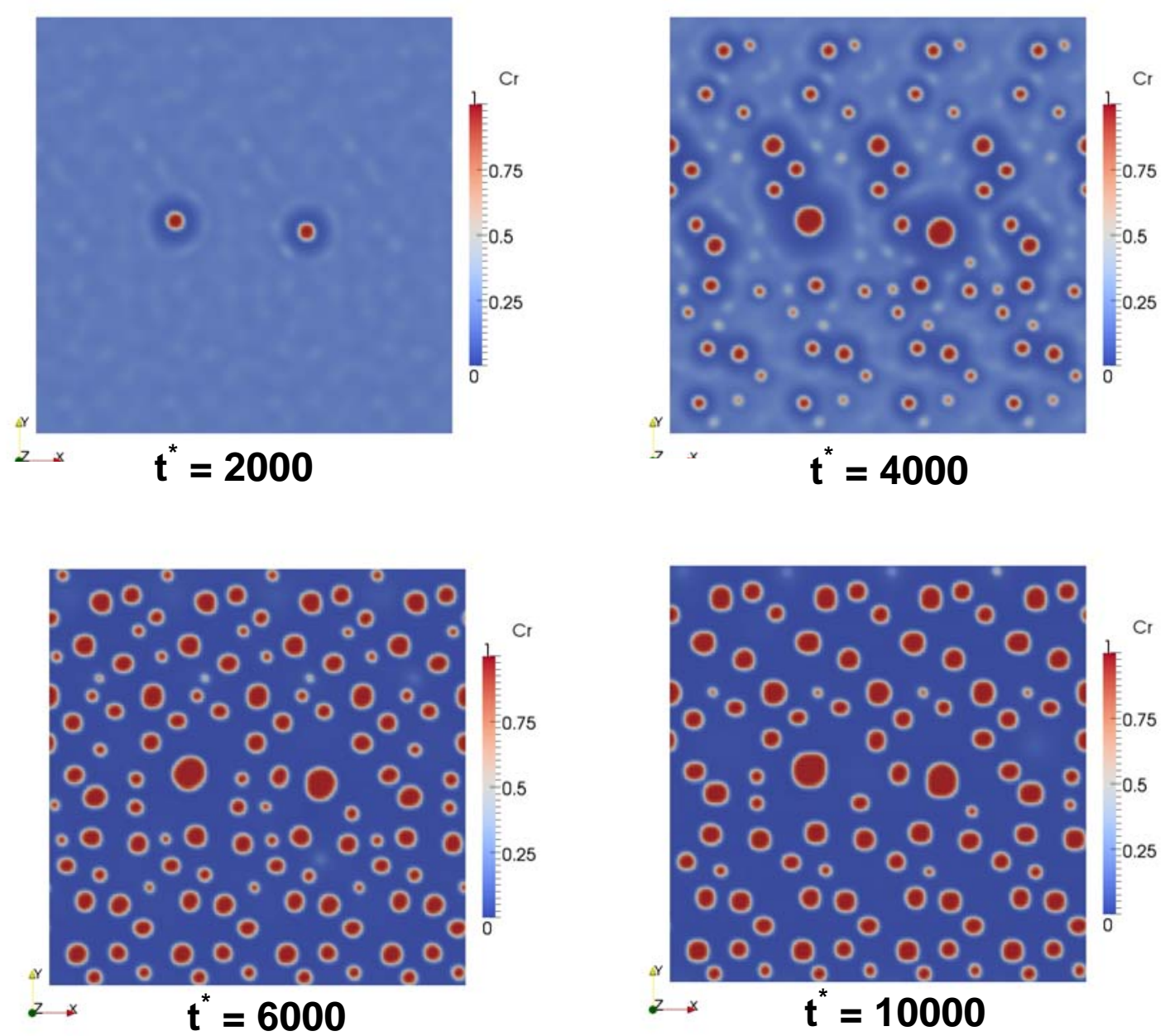

Figure 16. Microstructural evolution behavior of $\mathrm{Fe}-\mathrm{Cr}$ alloy with 20 at. $\% \mathrm{Cr}$ at $535 \mathrm{~K}$ with the presence of an edge dislocation dipole.

Also, the depletion of the local $\mathrm{Cr}$ concentration around the precipitates can be clearly inferred from the figure. Although the production of such curves yields statistical information regarding nucleation and growth kinetics of the aging process, it is not detailed in this study.

In the next set of simulations, we consider the microstructure evolution due to a dislocation dipole in the absence of any concentration fluctuations. For this case, the spatial and temporal evolution of the aging process is shown in Figure 18. As illustrated 
by the figure, the two precipitates first nucleate at the dislocations as described earlier. Up to the reduced time of 6000 , it appears these two are the only precipitates in the system. However, they have coarsened into a much larger size compared to their sizes at the reduced time of 2000. Of course, this coarsening behavior is related to the concentration flux driven by the stress gradients introduced by the dislocation dipole, which is completely different than the Oswald ripening seen earlier in Figures 3 and 4. At the reduced time of 8000 and 10000, we see the nucleation and growth of other nearby precipitates, resulting again from the concentration flux.
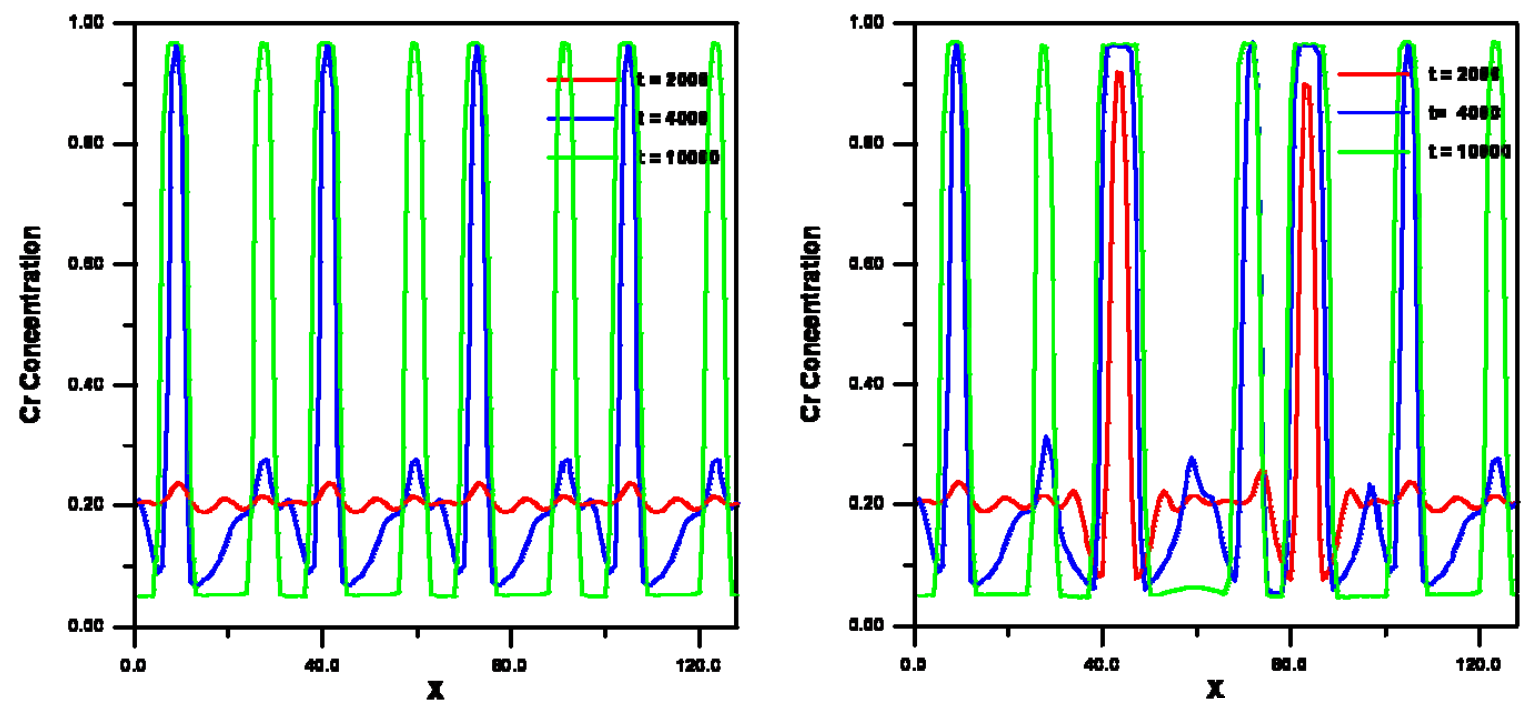

Figure 17. Variation of the concentration gradient with time along the line parallel to $\mathrm{x}_{1}$ direction and in the center of the simulation cell without the presence of dislocation (left) and with the presence of an edge dislocation dipole (right) for identical simulation conditions.

As previously indicated, if the dislocation dipole was not in the system and with the presence of no concentration fluctuation, the system would not evolve at all due to the absence of any intrinsic homogenous nucleation mechanism. 

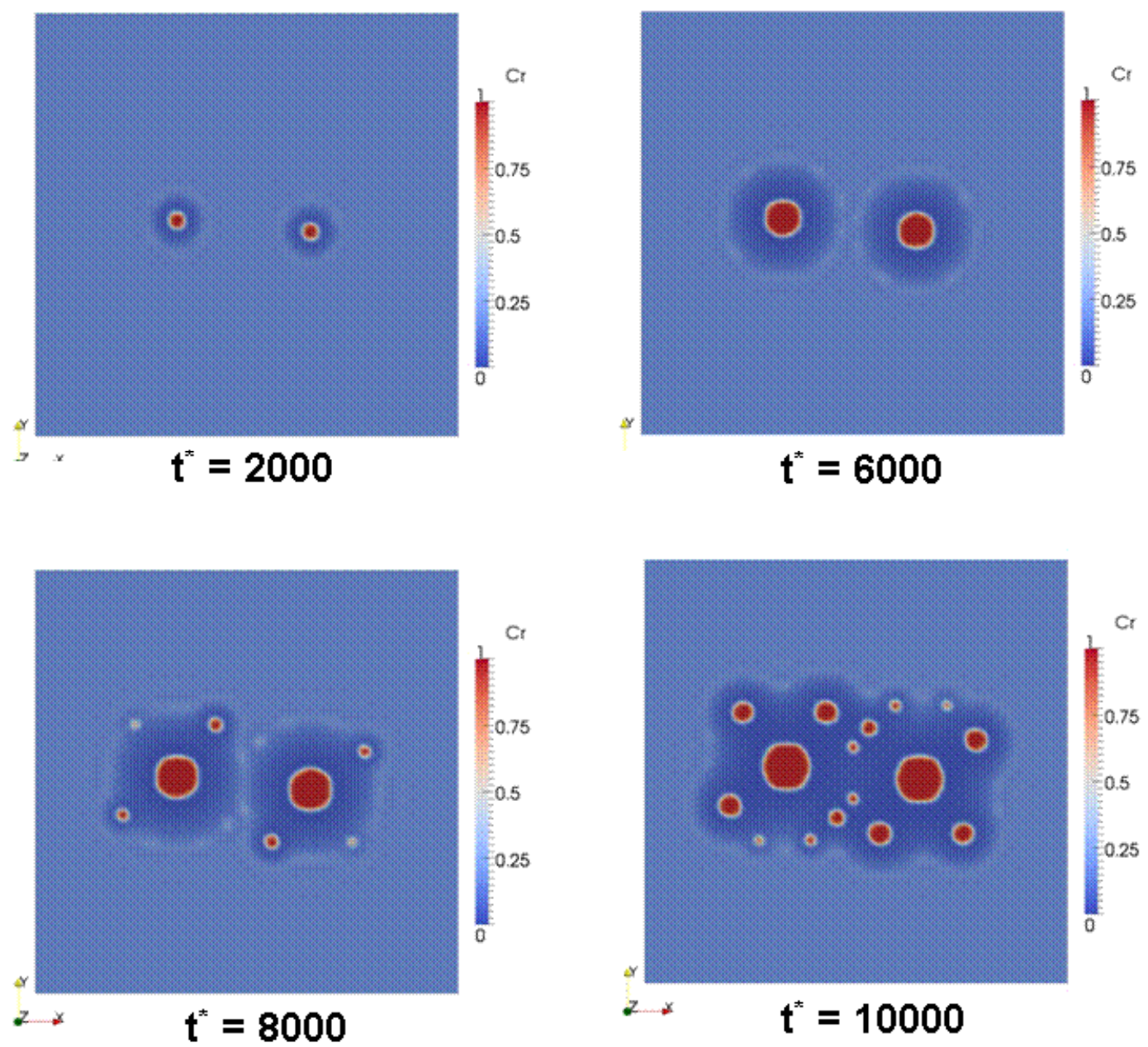

Figure 18. Microstructural evolution behavior of $\mathrm{Fe}-\mathrm{Cr}$ alloy with $20 \mathrm{at} . \% \mathrm{Cr}$ at $535 \mathrm{~K}$ with the presence of an edge dislocation dipole and without any compositional fluctuation in the system.

Next, the aging process due to the presence of the two dislocation walls, as summarized in Figure 19, is studied. Again in this simulation, there was no compositional inhomogeneity in the system, and the dislocation walls presented with a number of dislocation dipoles, separated 16 unit dimension apart from each other in the $\mathrm{x}_{2}$ direction (a tilt grain boundary representation). Again, the early nucleation and growth of the precipitates at the dislocations forming the walls can be seen from the figure (at reduced times of 2000 and 6000) with the heterogeneous nucleation and concentration flux 
mechanisms. Figure 19 also shows that with further growth (between the reduced time of 8000 and 10000), the formation of the platelet $\mathrm{Cr}$ precipitates takes place with coalescence compared to the circular ones seen earlier.
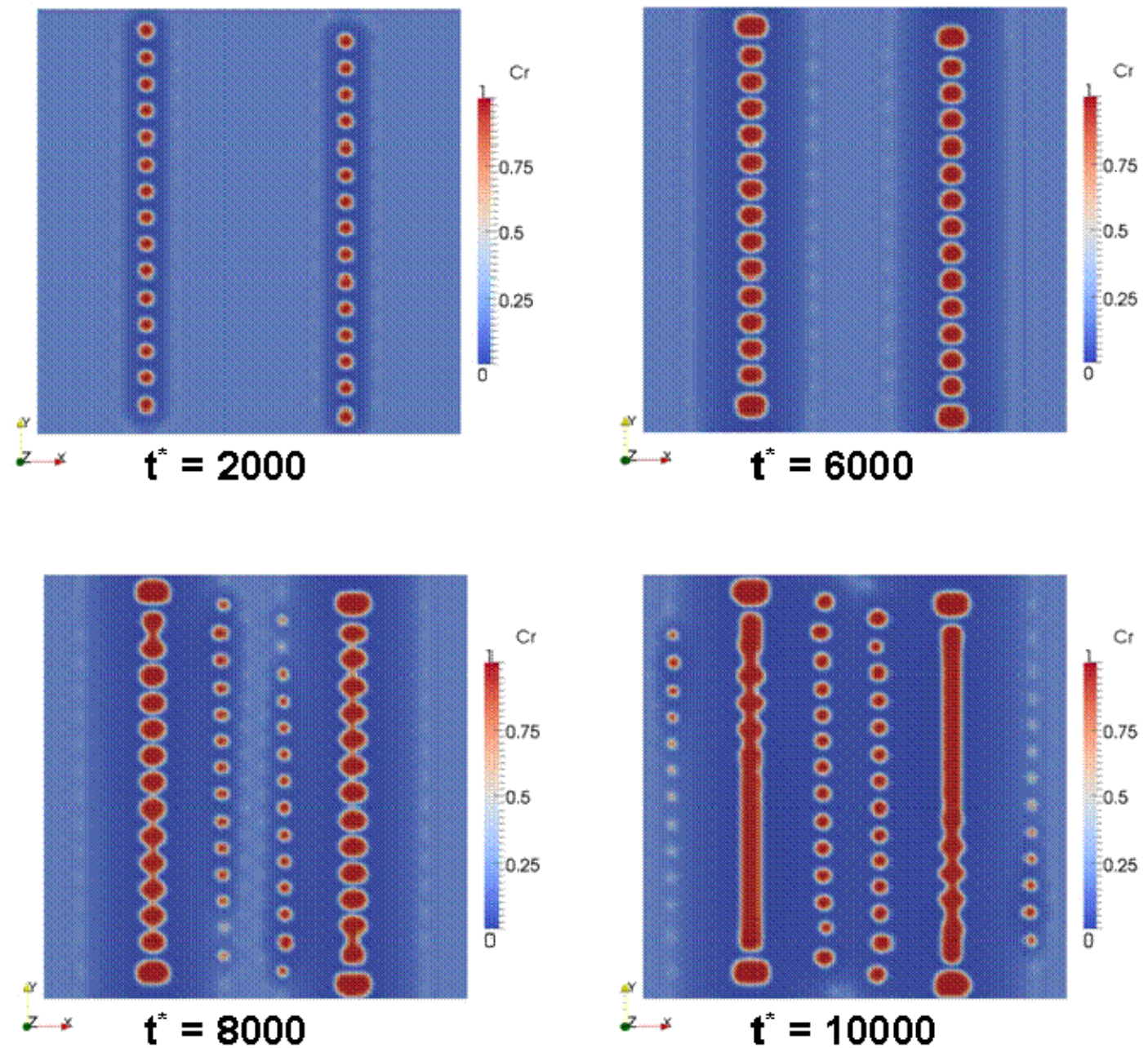

Figure 19. Microstructural evolution behavior of $\mathrm{Fe}-\mathrm{Cr}$ alloy with $20 \mathrm{at} \% \mathrm{Cr}$ at $535 \mathrm{~K}$ with the presence of two dislocation walls and without any compositional fluctuation in system.

From the results presented, the influence of underlying microstructural features -- in this case, simple dislocations dipoles and walls -- on the microstructure evolution is cleary demonstrated. As shown, they do not merely introduce a heterogeneous nucleation mechanism, but they also may alter the growth kinetics with completely different diffusional mechanisms and evolving morphology of the phases. 


\section{CONCLUDING REMARKS}

In this study, we developed a computational framework for homogenous and heterogenous nucleation mechanisms into the generic phase-field model. For homogenous nucleation, we proposed a general nucleation scheme to introduce the nuclei or concentration fluctuations into phase-field modeling. The scheme is based on nucleation probability determined by local supersatuation, diffusivity, and temperature. The thermodynamic and kinetic properties of $\mathrm{Fe}-\mathrm{Cr}$ alloys from atomistic simulations are used for quantitative simulations. Simulation results on nucleation rate and coarsening kinetics in two-dimensional models demonstrate the scheme is feasible. However, a quantitative assessment of nucleation theory in solids is inherently difficult. In the proposed nucleation scheme, there are two parameters, $\kappa_{1}$ and $\kappa_{2}$, which are functions of solute supersatuation, atomic impinging rates, interface area of the critical nucleus, atomic sites in the representative volume, temperature, and time. To further evaluate the nucleation scheme, a set of parametric studies for the effects of $\kappa_{1}$ and $\kappa_{2}$ on nucleation rates, as well as evolution of precipitate size distribution and comparison with experimental and atomic simulation results, are needed.

For the heteregoneous nucleation mechanism, we studied nucleation and growth behavior of $\mathrm{Cr}$ precipitates due to the presence of dislocations. In the model, the presence of the dislocations was accounted for via the eigenstrain formalism of edge dislocations in two dimensions. Even in its simple form, the results obtained from the simulations illustrate the importance of the underlying substructure of the microstructure (i.e., dislocations or grain boundaries) on the evolution behavior. In addition to obtaining relevant physical parameters (diffusion coefficients and mobility of the interfaces, formation energies, etc.), the realistic representation of the submicrostructure (dislocation entangles, junctions and jogs, and grain boundary morphologies, etc.) and associated co-evolution for more realistic and quantitative modeling is also challenging.

In this study, the phase-field simulations were conducted on fixed grids for both nucleation mechanisms. It is well established that both variable grids and time integration schemes offer significant computational advantages in phase-field modeling. The implementation of the nucleation algorithms developed in this study to the phasefield codes, as in MARMOT, that use such variable temporal and spatial integration schemes also needs be further explored. 


\section{REFERENCES}

1. T.W. Heo, et.al., Scripta Mat. 63, 8, (2010).

2. A. S Stukowski, et. al. Model. Sim. Mater. Sci. Eng, 17, 0000, (2009).

3. A. Caro et.al. JOM, 59, 50, (2007).

4. B. Sadigh et.al., (in press).

5. S.Lu et. al. Phys. Rev. B, 82, 195103, (2010).

6. S.Lu. et. al. Phys. Status. Solidi B. 248, 2087, (2011).

7. F. Soisson et. al. Phys. Rev. B, 62, 203, (2000).

8. T.P.C. Klaver et.al. Model. Sim. Mater. Sci. Eng. 18, 075004, (2010).

9. C. Bonny et. al. Phil. Mag. 12, 1724, (2011).

10. N. Saunders et. al. "Calphad Calculation of phase diagrams: A comprensive Guide", Pergamon press New York (1998).

11. W. Xiong et. al. Solid State Phenomena, 172-174, 1060, (2011).

12. G. Bonny et. al. J. Nuc. Mater., 385, 268, (2009).

13. P. Olsson et. al. J. Nuc. Mater. 321, 84, (2003).

14. J.W. Gibbs, The collected works: Thermodynamics: Vol.1. Longmans, Green \& Co. New York, 1928.

15. E. Clouet, ASM. Handbook Vol. 22A, Fundamentals of Modeling for Metals Processing, D.U. Furrer and S.L. Semiatin (Eds), p. 203.

16. J.B. Zeldovich, Acta Physicochimica URSS, 18, 1, (1943).

17. C-G Lee et. al. Mat. Trans Online JIM, 31, 255, (1990).

18. M.H. Mathon, et.a 1. J. Nuc. Mater. 312, 236, (2003).

19. Cahn, J. W. \& Hilliard, J Chem Phys 28, 258-267 (1958).

20. Leclaire, A. D., J Nucl Mater 69-70, 70-96 (1978).

21. Wong, K. L. at. al. J Nucl Mater 386, 227-230, doi:10.1016/j.jnucmat.2008.12.092 (2009).

22. Volmer, M. \& Weber, Z Phys Chem-Stoch Ve 119, 277-301 (1926).

23. Becker, R. \& Doring, W. Ann Phys-Berlin 24, 719-752 (1935).

24. Zeldovich, J. B. Physicochim Urs 18, 1-22 (1943).

25. Russell, K. C. Adv Colloid Interfac 13, 205-318 (1980).

26. Cahn, J. W. \& Hilliard, J Chem Phys 31, 688-699 (1959).

27. Zhang, L., Chen, L. Q. \& Du, Q. J Comput Phys 229, 6574-6584, doi:DOI 10.1016/j.jcp.2010.05.013 (2010).

28. Simmons, J. P., Shen, C. \& Wang, Scripta Mater 43, 935-942 (2000).

29. Lifshitz, I. M. \& Slyozov, V. V. J Phys Chem Solids 19, $35-50$ (1961).

30. Wagner, C. Z Elektrochem 65, 581-591 (1961).

31. Martinez, E., Fu, C. C., Levesque, M., Nastar, M. \& Soisson, Solid State Phenomen 172-174, 1016-1 (2011).

32. Y.S. Li, X.S. Li, T.Y. Zhang, J. Nuc. Mat, 395,120, (2009).

33. S.Y. Hu and L.Q. Chen, Acta. Mater., 49,463, (2001).

34. T. Mura, "Mechanics of defects in solids", Martinius Nijfoff publishers, (1982).

35. L.Q. Chen and J. Shen, Comp. Phys. Commun., 108, 147, (1998). 